\title{
Vinculación postcrisis entre empleo y esfuerzo \\ universitario: perspectiva comparada de \\ España y Canarias (1996-2017)
}

\author{
Llanos Castro, Ángel \\ Universidad de La Laguna, España \\ angel.Ilanoscastro@gmail.com
}

\section{Resumen}

La salida de la crisis económica se ha manifestado en España con una intensa creación de empleo durante los últimos 3 años (+2,1 millones). Para conocer el valor de la cultura del esfuerzo a la hora de obtener una educación superior, este trabajo analiza el contexto y la evolución reciente de la enseñanza universitaria en España y Canarias en perspectiva comparada con el resto de la Unión Europea y los países de la OCDE, así como los principales recursos públicos y privados existentes en la transición educativa hacia el empleo, detallándose los resultados más importantes de la gestión pública de las titulaciones superiores y sus efectos sobre el nivel de inclusión social de la población.

Mediante la metodología habitual de la Ciencia Política y la investigación social, se realiza un diseño diacrónico (1996-2017) en torno a cinco bloques: contexto y evolución; recursos humanos y económicos; escolarización; procesos educativos; y resultados. La discusión se estructura mediante un análisis longitudinal-temporal y transversal-comparativo de las variables estadísticas oficiales más destacadas sobre la educación superior, en base a la posición teórica previamente establecida.

Las conclusiones más importantes indican luces y sombras en el panorama universitario canario, y una estrecha vinculación en España entre la disposición de titulación superior y la ocupación de los nuevos puestos de trabajo creados desde 2014, así como el paulatino abandono de la formación continua entre los ocupados desde la salida de la recesión y ciertas dificultades estructurales en la universidad pública española.

\section{Abstract}

The exit from the economic crisis has manifested itself in Spain with an intense job creation during the last 3 years (+2.1 million). In order to know the value of the culture of effort when obtaining a higher education, this paper analyzes the context and the recent evolution of university education in Spain and the Canary Islands in perspective compared to the rest of the European Union and the countries of the OECD, as well as the main public and private resources existing in the educational transition to employment, detailing the most important results of public management of higher degrees and their effects on the level of social inclusion of the population. Through the usual methodology of Political Science and social research, a diachronic design (1996-2017) is carried out around five blocks: context and evolution; human and economic resources; schooling; educational processes; and results. The discussion is structured through a longitudinal-temporal and cross-comparative analysis of the most important official statistical variables on higher education, based on the previously established theoretical position.

The most important conclusions indicate lights and shadows in the Canarian university panorama, and a close link in Spain between the provision of higher qualification and the occupation of the new jobs created since 2014, as well as the gradual abandonment of continuing education among the occupied since the end of the recession and certain structural difficulties in the Spanish public university.)

Palabras clave: educación universitaria, gasto público, población ocupada, cultura del esfuerzo.

Keywords: university education, public expenditure, employed population, culture of effort.

\section{INTRODUCCIÓN}

La salida de la crisis de 2008 se ha manifestado en España con una intensa creación de empleo hasta llegar a 2,1 millones de personas ocupadas más en septiembre de 2017 que en marzo de 2014. En los países OCDE, la disponibilidad de estudios superiores (Licenciatura, Grado, Máster Oficial, Doctorado o equivalente), es sinónimo de una mayor empleabilidad, mejor retribución y menor tasa de desempleo. Pero en Canarias, aunque el descenso del paro también ha sido significativo, aún existen índices muy superiores a la media del resto del Estado. 
Esta comunicación se plantea como objetivo general conocer el valor de la cultura del esfuerzo al obtener una educación superior, tanto a nivel global para el conjunto de España como específicamente en el caso canario. Para ello, la investigación pretende alcanzar los siguientes objetivos específicos:

a. Analizar el contexto de la enseñanza universitaria en España y Canarias, en perspectiva comparada con el resto de Europa y los países de la OCDE.

b. Valorar los principales recursos existentes en el camino de la transición educativa hacia el empleo.

c. Y concretar los resultados más importantes alcanzados.

El tema seleccionado es de interés ante la magnitud de la estructura pública y privada que moviliza la educación universitaria, y también por el fenómeno «ni-ni», con el que llegó a estereotiparse a un colectivo genérico de jóvenes cuando sus integrantes no son un grupo «homogéneo» sino jóvenes «de entre 20 a 25 años» que «revelan un estatus de fragilidad psicosocial peculiar, únicamente comprensible desde los imaginarios posmodernos sobre el trabajo, la supervivencia y el consumo» (INJUVE, 2011: 96).

La metodología utilizada ha sido la habitual de la Ciencia Política y la investigación social; en concreto, el análisis cuantitativo y cualitativo de las fuentes seleccionadas: documentos institucionales, textos y manuales pedagógicos, y estadísticas oficiales. En las tablas se han eliminado datos innecesarios a fin de mostrar una mayor claridad expositiva, y se han incluido nuevas fórmulas comparativas.

En el establecimiento del período de estudio ha primado la obtención de los datos más recientes (algunos de septiembre de 2017), junto con una visión comparada desde 2005; aunque el ámbito temporal se extiende dos décadas para ciertas variables. La mayor dificultad ha sido la selección de las fuentes bibliográficas y la búsqueda de los estadísticos más ilustrativos; y el límite lo ha marcado su propia disponibilidad. Este tipo de recursos han aportado ventajas, ya que facilitan una información no reactiva al no afectarle relación alguna entre quien los analiza y los sujetos estudiados (no son distorsionados por sus efectos), permiten un análisis diacrónico y no requiere financiación al ser de libre acceso (a diferencia de los trabajos de campo).

\section{DISCUSIÓN}

\subsection{Contexto}

El debate que surge en periodos de crisis en torno a los problemas de la educación, impide valorar el crecimiento educativo durante el periodo democrático. Una expansión de la educación reglada de tal magnitud que «nunca hasta ahora había habido en España tal cantidad de ciudadanos con un nivel formativo tan alto (...)» y «nunca hasta ahora un número tan elevado de españoles dedicaba en nuestro país tanto tiempo de sus vidas al aprendizaje formalizado»; así como «nunca hasta ahora la enseñanza universitaria había acogido a tantos estudiantes en su seno» (Requena y Bernardi, 2008: 242). Y eso pese a que en los periodos de bonanza económica, «la estrecha asociación existente entre población ocupada en categorías de baja cualificación laboral y las tasas nacionales de desescolarización a los 18 años» estimulan una «inserción laboral prematura» en «trabajos poco cualificados» para "los jóvenes con menores expectativas académicas», conjugándose una triple situación de «elevados niveles de descualificación entre jóvenes ocupados, elevados niveles de déficit instructivo en la población adulta y elevados niveles de abandono educativo temprano» (Alegre, M., y Subirats, J., 2016: 357). Desde la óptica de la estratificación social, se ha pasado del escenario existente en buena parte del siglo XX, caracterizado por una «fuerte asociación entre el logro educativo y la procedencia social y una desigual distribución de los títulos educativos entre las distintas clases», a una "reducción de las desigualdades clasistas de oportunidades educativas» (Requena, Salazar y Radl, 2013: 210-213). 
Para contextualizar el periodo analizado, se han seleccionado cuatro ítems:

1. Se ha producido un escenario de intensa creación de empleo en España entre 2014 y 2017, y que se resume en dos datos:

a. La Encuesta de Población Activa situaba en marzo de 2014 a 16.950.600 personas ocupadas; cifra que se elevó hasta los 19.049.200 en septiembre de 2017, lo que supone un incremento de 2.098.600 ocupados en tres años y medio (INE).

b. La Seguridad Social pasó de contar con 16.305 .000 afiliados en septiembre de 2013, a 18.460.200 en diciembre de 2017, por lo que los cotizantes se han incrementado en 2.155.200 personas en cuatro años (Tesorería General de la Seguridad Social).

2. En cuanto al acceso a la enseñanza superior de los jóvenes, España está por debajo de la tasa de la Unión Europea ya que un $47 \%$ de ellos iniciará un grado y un $9 \%$ un máster oficial, cuando la media comunitaria está situada en un $54 \%$ y un $23 \%$, respectivamente (CRUE, 2017: 8). Pero los resultados de los estudios superiores son mejores en España, pues la tasa de graduación en el año 2014 fue del 59,3\%, mientras que la media de la OCDE era del 49,1\% y la de la UE22 del 44,9\% (Ministerio de Educación, Cultura y Deporte, 2016b: 20). España tiene mejor posición que su contexto internacional en el número medio de alumnos por profesor universitario, ya que su ratio está en 13 mientras que en la OCDE y la UE22 es de 17 alumnos por profesor (ibídem, 54). Y la nota media de los egresados en España del curso 2013-2014 fue de 7,29, y 8,17 entre los que finalizaron un Máster oficial (Ministerio de Educación, Cultura y Deportes, 2016a: 43).

2. En 2015 , el $36 \%$ de la población de entre 25 y 64 años de los países OCDE disponían de estudios superiores, un punto más que la media española (OCDE, 2016: 41). Pero España es el quinto país de la UE con los precios públicos universitarios más elevados (CRUE, 2017: 30), con un coste medio de 1.991 euros para la matrícula de máster oficial y de 1.110 euros para los grados; tan sólo por detrás de Irlanda, Reino Unido, Holanda e Italia, y muy lejos de los 0 euros que cuesta la matrícula de Grado y Máster en Alemania, Austria, Noruega, Suecia, Finlandia y Dinamarca. Según el 'Observatorio de las Ocupaciones 2016' (Servicio Público de Empleo Estatal, 2016: 83), las cinco principales contrataciones de los españoles entre 16 y 29 años en 2015 fueron: camareros (991.652 personas), vendedores en tiendas (470.209 personas), peones agrícolas (459.150), peones de industrias manufactureras (414.173) y personal de limpieza (212.119 jóvenes).

Una primera aproximación muestra un escenario de fuerte creación de nuevos puestos de trabajo, con luces y sombras en cuanto a los resultados de España en la educación universitaria en comparación al resto de Europa, y un empleo juvenil con predominio de puestos de baja cualificación. Este contexto se completa con tres referencias a Canarias:

1. El Gasto en I+D en 2014 de Canarias sobre el global del PIB fue del 0,46\%, tan sólo por delante de Baleares $(0,32 \%)$ y por debajo del $1,23 \%$ del total español (Ministerio de Educación, Cultura y Deportes, 2016a: 153).

2. Las islas, con un PIB per cápita de 19.900 euros en el periodo 2014-15 (Tabla-1), están por encima de Murcia, Castilla-La Mancha, Andalucía y Extremadura. La población canaria de 25 a 64 años con estudios post-obligatorios era del $50,7 \%$, casi siete puntos menos que la media española; y la tasa bruta de graduados universitarios alcanzaba el 28,3 , aún más lejos de la media nacional $(63,8)$.

3. Pese a que las mujeres canarias con titulación superior es superior a los hombres en cinto puntos (Tabla-2), no son comparables al resto de las autonomías, pues sólo la Región de Murcia $(28,8 \%)$ tiene un porcentaje de universitarias inferior a Canarias $(29,9 \%)$, situándose ambas a la cola del país.

El análisis contextual de Canarias la sitúa entre las regiones más pobres de España, con menor gasto en I+D y entre las últimas en el porcentaje de graduados universitarios, con una comparación especialmente negativa en el caso de las mujeres. 
Tabla 1. La educación y el contexto (2014-15).

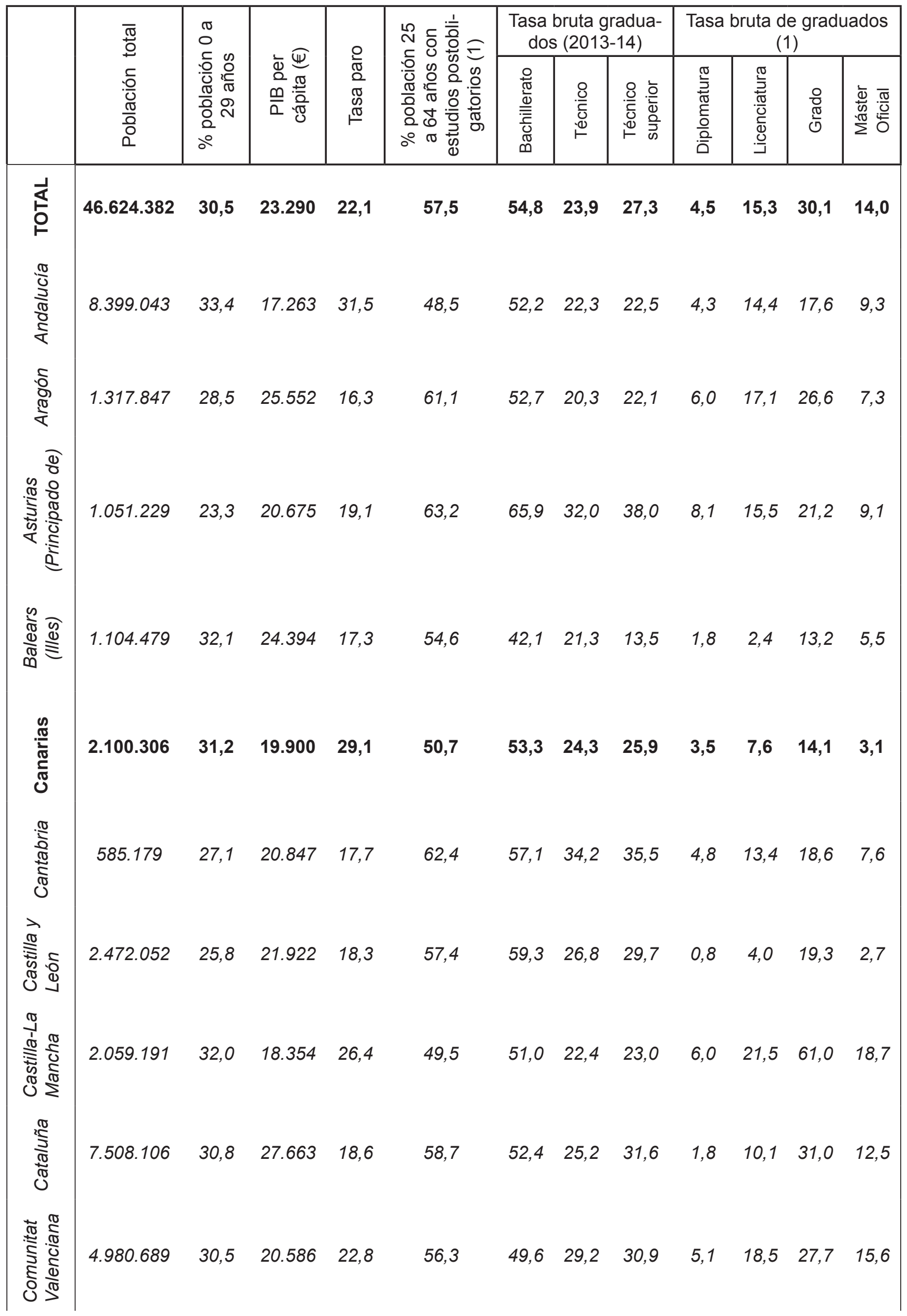




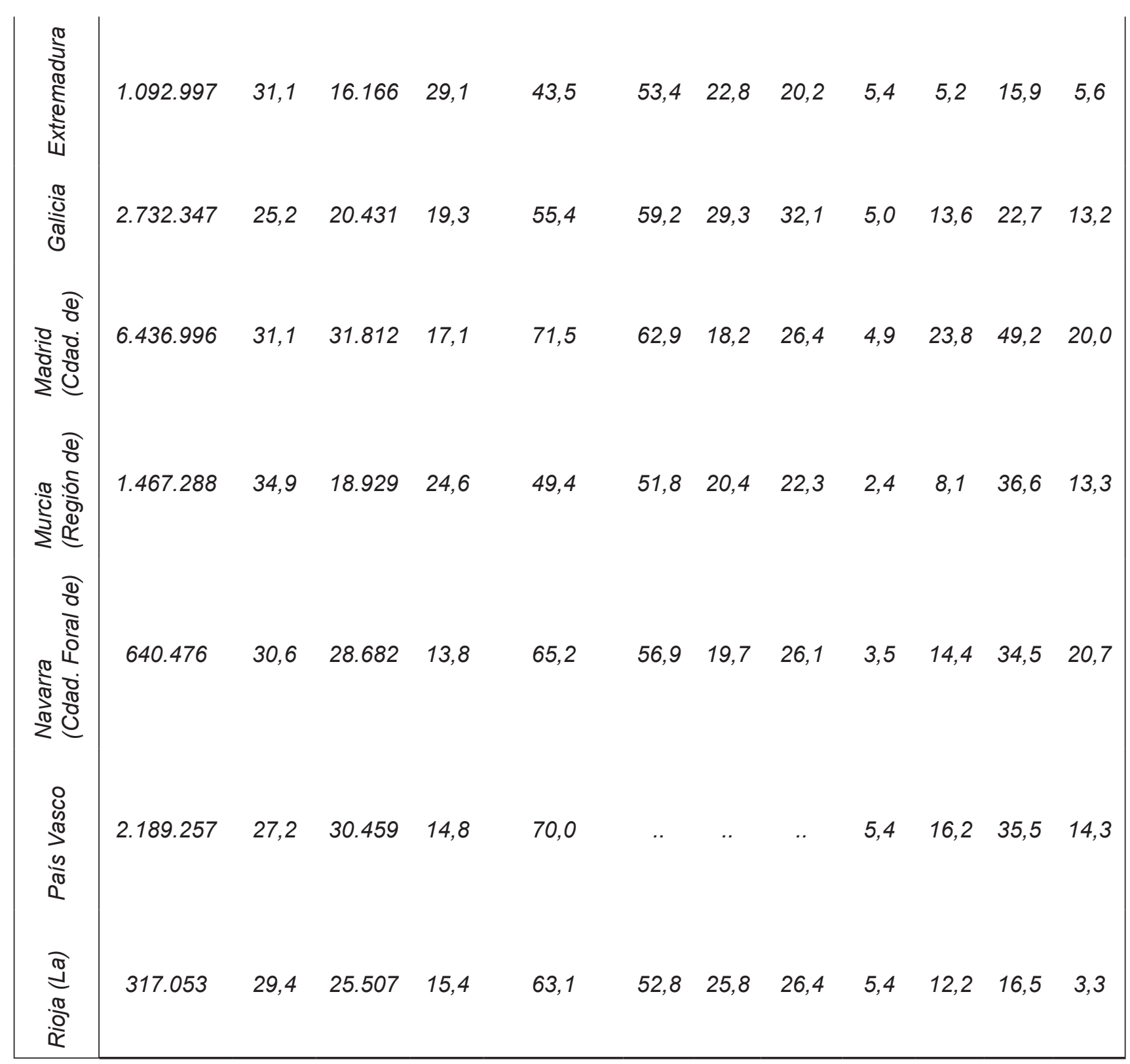

Fuente: Ministerio de Educación, Cultura y Deporte.

(1) Los titulados universitarios se han clasificado por ubicación del rectorado de la universidad (Andalucía engloba Ceuta y Melilla, y C. Madrid a Guadalajara).

No se incluyen Ceuta y Melilla por su escasa representatividad. 
Tabla 2. Nivel de formación de la población de 25 a 65 años en el año 2015 (1) (2).

\begin{tabular}{|c|c|c|c|c|c|c|c|c|c|}
\hline & \multicolumn{3}{|c|}{ AMBOS SEXOS } & \multicolumn{3}{|c|}{ HOMBRES } & \multicolumn{3}{|c|}{ MUJERES } \\
\hline & 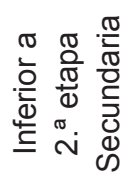 & 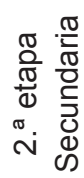 & 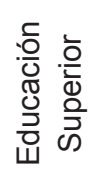 & 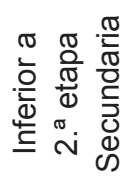 & 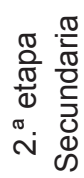 & 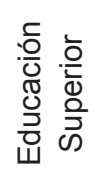 & 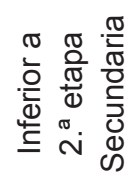 & 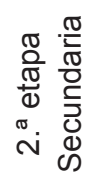 & $\begin{array}{c}\text { Educación } \\
\text { Superior }\end{array}$ \\
\hline Total & 42,6 & 22,4 & 35,1 & 44,7 & 22,7 & 32,7 & 40,4 & 22,0 & 37,5 \\
\hline Andalucía & 51,4 & 20,2 & 28,3 & 53,7 & 20,0 & 26,3 & 49,2 & 20,5 & 30,3 \\
\hline Aragón & 39,0 & 25,5 & 35,6 & 40,9 & 26,1 & 33,0 & 37,0 & 24,8 & 38,2 \\
\hline $\begin{array}{c}\text { Asturias } \\
\text { (Principado } \\
\text { de) }\end{array}$ & 36,7 & 23,6 & 39,6 & 39,5 & 23,3 & 37,2 & 34,0 & 23,9 & 42,0 \\
\hline $\begin{array}{c}\text { Balears } \\
\text { (Illes) }\end{array}$ & 45,4 & 27,4 & 27,2 & 48,8 & 27,3 & 23,9 & 41,9 & 27,4 & 30,7 \\
\hline Canarias & 49,3 & 24,1 & 26,6 & 52,1 & 23,6 & 24,3 & 46,6 & 24,6 & 28,9 \\
\hline Cantabria & 37,6 & 24,3 & 38,1 & 40,0 & 24,0 & 36,0 & 35,3 & 24,6 & 40,1 \\
\hline $\begin{array}{l}\text { Castilla y } \\
\text { León }\end{array}$ & 42,5 & 22,7 & 34,7 & 46,1 & 23,3 & 30,6 & 38,8 & 22,2 & 39,0 \\
\hline $\begin{array}{l}\text { Castilla- } \\
\text { La Mancha }\end{array}$ & 50,5 & 22,0 & 27,5 & 53,0 & 21,2 & 25,7 & 47,7 & 22,9 & 29,4 \\
\hline Cataluña & 41,2 & 21,2 & 37,5 & 43,2 & 22,4 & 34,4 & 39,3 & 20,1 & 40,6 \\
\hline $\begin{array}{l}\text { Comunitat } \\
\text { Valenciana }\end{array}$ & 43,7 & 23,8 & 32,5 & 45,8 & 24,0 & 30,2 & 41,6 & 23,5 & 34,8 \\
\hline Extremadura & 56,5 & 17,0 & 26,5 & 60,1 & 16,2 & 23,7 & 52,7 & 17,8 & 29,4 \\
\hline Galicia & 44,7 & 20,2 & 35,2 & 47,2 & 20,6 & 32,1 & 42,1 & 19,7 & 38,1 \\
\hline
\end{tabular}




\begin{tabular}{|c|c|c|c|c|c|c|c|c|c|}
\hline $\begin{array}{c}\text { Madrid } \\
\text { (Comunidad } \\
\text { de })\end{array}$ & 28,5 & 24,6 & 46,9 & 29,2 & 25,7 & 45,1 & 27,8 & 23,6 & 48,5 \\
\hline $\begin{array}{c}\text { Murcia } \\
\text { (Región de) }\end{array}$ & 50,6 & 21,5 & 27,9 & 52,0 & 21,1 & 26,9 & 49,2 & 22,0 & 28,8 \\
\hline $\begin{array}{l}\text { Navarra } \\
\text { (Cdad. } \\
\text { Foral de) }\end{array}$ & 34,8 & 22,9 & 42,3 & 36,1 & 23,4 & 40,5 & 33,4 & 22,5 & 44,2 \\
\hline País Vasco & 30,1 & 22,2 & 47,8 & 30,3 & 23,0 & 46,8 & 29,8 & 21,4 & 48,8 \\
\hline Rioja (La) & 37,0 & 23,8 & 39,3 & 39,5 & 23,7 & 36,8 & 34,4 & 23,9 & 41,8 \\
\hline
\end{tabular}

Fuente: Ministerio de Educación, Cultura y Deporte.

(1) Los resultados están basados en medias anuales de datos trimestrales.

(2) Los datos deben ser tomados con precaución, pues los de tamaños muestrales pequeños están afectados por fuertes errores de muestreo.

No se incluyen Ceuta y Melilla por su escasa representatividad. 


\subsection{Análisis longitudinal-temporal y transversal-comparativo}

I.- Los recursos. El sector universitario español, aunque con una creciente oferta privada, es eminentemente público: de las 81 universidades, 50 son públicas y 31 privadas (Tabla-3).

Tabla 3. Clasificación de los centros por tipo y titularidad (2014-15).

\begin{tabular}{|c|c|c|c|c|c|c|}
\hline & \multicolumn{2}{|c|}{ TODOS LOS CENTROS } & \multicolumn{2}{|c|}{ CENTROS PÚBLICOS } & \multicolumn{2}{|c|}{ CENTROS PRIVADOS } \\
\hline & 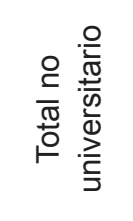 & $\begin{array}{l}0 \\
\frac{0}{0} \\
\frac{\pi}{0} \\
\frac{0}{0} \\
\frac{0}{0} \\
\stackrel{\geq}{5}\end{array}$ & 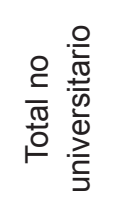 & $\begin{array}{l}0 \\
\frac{0}{0} \\
\frac{\pi}{0} \\
\frac{0}{0} \\
\frac{0}{0} \\
\stackrel{2}{1} \\
\frac{1}{5}\end{array}$ & 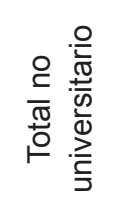 & $\begin{array}{l}0 \\
\frac{0}{0} \\
\frac{\pi}{0} \\
\frac{0}{0} \\
\frac{0}{0} \\
\stackrel{\geq}{5} \\
\end{array}$ \\
\hline Total & 31.430 & 81 & 21.839 & 50 & 9.591 & 31 \\
\hline Andalucía & 6.669 & 11 & 4.589 & 10 & 2.080 & 1 \\
\hline Aragón & 1.011 & 2 & 725 & 1 & 286 & 1 \\
\hline Asturias (Principado de) & 543 & 1 & 426 & 1 & 117 & 0 \\
\hline Balears (Illes) & 601 & 1 & 409 & 1 & 192 & 0 \\
\hline Canarias & 1.219 & 3 & 956 & 2 & 263 & 1 \\
\hline Cantabria & 333 & 2 & 233 & 1 & 100 & 1 \\
\hline Castilla y León & 1.599 & 9 & 1.277 & 4 & 322 & 5 \\
\hline Castilla-La Mancha & 1.604 & 1 & 1.274 & 1 & 330 & 0 \\
\hline Cataluña & 5.217 & 12 & 3.685 & 7 & 1.532 & 5 \\
\hline Comunitat Valenciana & 3.063 & 9 & 1.991 & 5 & 1.072 & 4 \\
\hline Extremadura & 968 & 1 & 854 & 1 & 114 & 0 \\
\hline Galicia & 2.068 & 3 & 1.474 & 3 & 594 & 0 \\
\hline Madrid (Comunidad de) & 3.630 & 14 & 1.895 & 6 & 1.735 & 8 \\
\hline Murcia (Región de) & 855 & 3 & 631 & 2 & 224 & 1 \\
\hline $\begin{array}{c}\text { Navarra } \\
\text { (Comunidad Foral de) }\end{array}$ & 481 & 2 & 380 & 1 & 101 & 1 \\
\hline País Vasco & 1.291 & 3 & 849 & 1 & 442 & 2 \\
\hline Rioja (La) & 203 & 2 & 135 & 1 & 68 & 1 \\
\hline Univ. especiales & - & 2 & - & 2 & - & 0 \\
\hline
\end{tabular}

Fuente: Ministerio de Educación, Cultura y Deporte.

No se incluyen Ceuta y Melilla por su escasa representatividad.

Pero estos términos absolutos se relativizan al descender al interior de los centros y analizar la tipología del profesorado. En Canarias, frente a 3.262 profesores que prestaban sus servicios en el 
curso 2014/15 en las dos universidades públicas (La Laguna y Las Palmas de Gran Canaria), apenas 82 docentes lo hacían en universidades privadas (Tabla-4). Esta testimonial presencia docente en centros privados universitarios, contrasta con la importante participación de los centros privados en la educación de régimen general no universitaria, pues frente a los 21.457 profesores con los que cuentan los centros públicos de Canarias, 5.692 trabajan en centros privados (Tabla-4).

Tabla 4. Profesorado por tipo de centro 2014-15.

\begin{tabular}{|c|c|c|c|c|c|c|c|c|c|c|c|c|c|c|c|}
\hline & \multicolumn{5}{|c|}{ TODOS LOS CENTROS } & \multicolumn{5}{|c|}{ CENTROS PÚBLICOS } & \multicolumn{5}{|c|}{ CENTROS PRIVADOS } \\
\hline & $\begin{array}{l}\vec{\leftarrow} \\
\stackrel{5}{0} \\
\stackrel{1}{1}\end{array}$ & 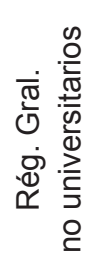 & 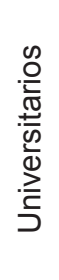 & 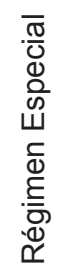 & 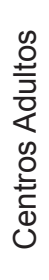 & 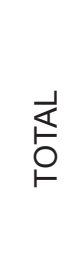 & 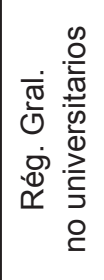 & 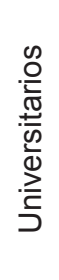 & 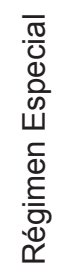 & 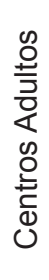 & $\begin{array}{l}\frac{1}{5} \\
\stackrel{0}{0}\end{array}$ & 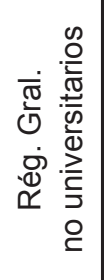 & 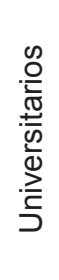 & 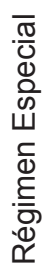 & 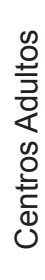 \\
\hline $\begin{array}{l}\bar{\pi} \\
\stackrel{\pi}{0}\end{array}$ & $\begin{array}{l}\widetilde{N} \\
\mathscr{\Omega} \\
0 \\
0 \\
\infty\end{array}$ & 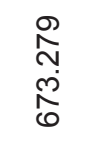 & 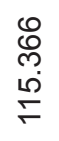 & $\begin{array}{l}\stackrel{\infty}{\infty} \\
\stackrel{\infty}{\infty} \\
\infty \\
\infty\end{array}$ & $\begin{array}{l}\stackrel{8}{0} \\
\stackrel{0}{\circ}\end{array}$ & $\begin{array}{l}0 \\
\stackrel{\sim}{0} \\
\infty \\
\infty \\
\sigma\end{array}$ & 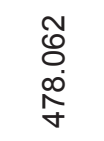 & \begin{tabular}{l}
$\infty$ \\
$\stackrel{\infty}{0}$ \\
\multirow{\infty}{\infty}{}
\end{tabular} & 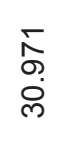 & $\frac{\stackrel{\rho}{+}}{\stackrel{\sim}{\sigma}}$ & $\begin{array}{l}0 \\
\stackrel{0}{\Lambda} \\
\infty \\
\frac{\infty}{N}\end{array}$ & $\begin{array}{l}\hat{N} \\
\stackrel{N}{ } \\
\stackrel{-}{\sigma}\end{array}$ & 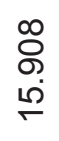 & Nָ & ఫ్ల \\
\hline $\begin{array}{l}\frac{\frac{\pi}{0}}{\mathrm{O}} \\
\frac{\bar{\sigma}}{\mathrm{T}} \\
\frac{\mathrm{C}}{\mathrm{L}}\end{array}$ & $\begin{array}{l}\mathscr{N} \\
\infty \\
\infty \\
0 \\
0 \\
\infty\end{array}$ & 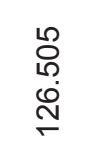 & $\begin{array}{l}\infty \\
\stackrel{+}{\sigma} \\
\dot{\varphi}\end{array}$ & 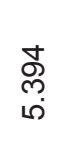 & 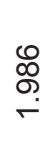 & 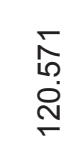 & $\begin{array}{l}\stackrel{ల}{ } \\
\infty \\
\dot{\infty}\end{array}$ & $\begin{array}{l}\frac{10}{\infty} \\
\dot{0} \\
\dot{0}\end{array}$ & $\begin{array}{l}\overline{\mathscr{g}} \\
\stackrel{+}{\forall}\end{array}$ & 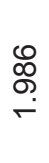 & 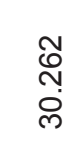 & $\begin{array}{l}8 \\
\mathbb{8} \\
\stackrel{N}{ }\end{array}$ & $\stackrel{m}{m}$ & 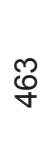 & 0 \\
\hline $\begin{array}{l}.0 \\
\stackrel{0}{0} \\
\frac{\pi}{<}\end{array}$ & 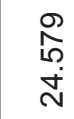 & $\begin{array}{l}\text { ठे } \\
\infty \\
\infty \\
\infty\end{array}$ & $\begin{array}{l}\text { స్ల } \\
\text { }\end{array}$ & $\stackrel{ }{\stackrel{ }{ }}$ & N & $\begin{array}{l}\infty \\
\stackrel{\circ}{ } \\
\infty \\
\infty\end{array}$ & $\begin{array}{l}\stackrel{+}{0} \\
\infty \\
\stackrel{M}{\sim}\end{array}$ & 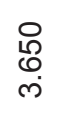 & న్ & $\underset{\mathscr{o}}{\mathscr{O}}$ & $\begin{array}{l}\overline{\hat{\sigma}} \\
0 \\
10\end{array}$ & $\begin{array}{l}8 \\
\text { Oे } \\
\text { in }\end{array}$ & 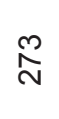 & $\stackrel{\infty}{\sim}$ & ৪ \\
\hline 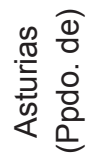 & $\begin{array}{l}\stackrel{N}{\Omega} \\
\stackrel{6}{6}\end{array}$ & 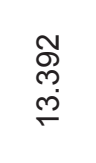 & 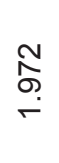 & ర్ల & $\stackrel{\circ}{\circ}$ & $\stackrel{\stackrel{\mathscr{N}}{ }}{\stackrel{\sim}{\sim}}$ & $\begin{array}{l}8 \\
\stackrel{\leftrightarrow}{0} \\
\stackrel{0}{\circ}\end{array}$ & 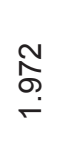 & $\widehat{\widehat{D}}$ & $\stackrel{8}{\circ}$ & $\begin{array}{l}\hat{N} \\
\text { ஸे }\end{array}$ & $\begin{array}{l}\stackrel{Q}{N} \\
\stackrel{\text { N }}{\text { N }}\end{array}$ & 0 & 흠 & 0 \\
\hline 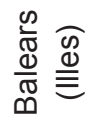 & 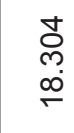 & $\begin{array}{l}\infty \\
\ddot{O} \\
\stackrel{\varphi}{\varphi}\end{array}$ & 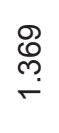 & $\frac{\pi}{6}$ & $\stackrel{m}{\sim}$ & 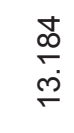 & 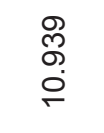 & $\begin{array}{l}\stackrel{8}{0} \\
\text { లా } \\
\stackrel{-}{ }\end{array}$ & ஜ & $\stackrel{m}{\sim}$ & $\frac{\stackrel{O}{\leftarrow}}{i \circ}$ & $\begin{array}{l}\text { क् } \\
\text { ठ } \\
\text { ம் }\end{array}$ & 0 & $\bar{\sim}$ & 0 \\
\hline 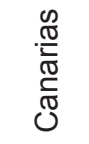 & $\begin{array}{l}\underset{N}{N} \\
\text { స్ }\end{array}$ & 竞 & 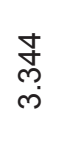 & $\underset{r}{\stackrel{\gamma}{\sigma}}$ & ஜ̊ & 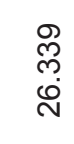 & 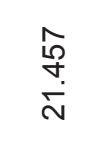 & $\begin{array}{l}\text { đै } \\
\stackrel{\text { m }}{ }\end{array}$ & $\stackrel{\vec{m}}{r}$ & \&্ল & $\begin{array}{l}\widehat{N} \\
\text { co } \\
\text { in }\end{array}$ & $\begin{array}{l}\text { هે } \\
\text { ம் } \\
\text { ம் }\end{array}$ & ๗ & 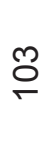 & 0 \\
\hline $\begin{array}{l}\frac{\pi}{2} \\
\frac{0}{0} \\
\stackrel{\pi}{\pi} \\
\frac{\pi}{\pi} \\
0\end{array}$ & $\begin{array}{l}\text { 寺 } \\
\text { Oे }\end{array}$ & $\begin{array}{l}\infty \\
\stackrel{\infty}{\$} \\
\infty\end{array}$ & 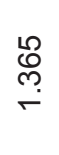 & $\widehat{\stackrel{m}{m}}$ & $\stackrel{\widehat{m}}{r}$ & $\begin{array}{l}\infty \\
\underset{\infty}{\infty} \\
\infty\end{array}$ & $\begin{array}{l}\text { Oे } \\
\text { bु } \\
0\end{array}$ & $\stackrel{\text { }}{\text { m}}$ & $\stackrel{0}{N}$ & $\stackrel{\widehat{m}}{\leftarrow}$ & 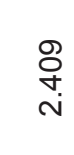 & 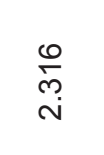 & స్ & $\overline{0}$ & 0 \\
\hline 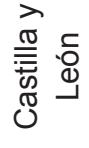 & $\begin{array}{l}\hat{b} \\
0 \\
\dot{f}\end{array}$ & $\begin{array}{l}\text { ठ } \\
\text { లి } \\
\text { ஸें }\end{array}$ & 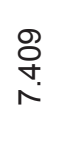 & \begin{tabular}{l}
$\hat{O}$ \\
\multirow{i}{N}{} \\
in
\end{tabular} & $\widehat{\hat{B}}$ & 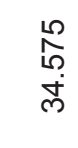 & 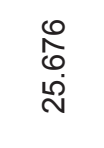 & 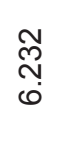 & 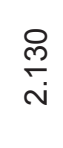 & గి & $\begin{array}{l}\text { o } \\
\text { o } \\
\stackrel{0}{\circ}\end{array}$ & 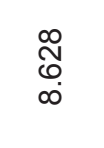 & 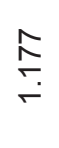 & $\stackrel{\hat{N}}{ }$ & 0 \\
\hline 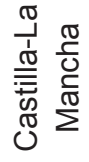 & 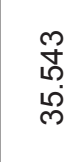 & $\frac{N}{\tilde{L}}$ & ্ָণ্ণ & $\begin{array}{l}\mathscr{8} \\
\stackrel{0}{\circ} \\
-\end{array}$ & กำ & $\begin{array}{l}\text { D. } \\
\text { مُ }\end{array}$ & $\begin{array}{l}\infty \\
\stackrel{0}{0} \\
\stackrel{N}{N}\end{array}$ & ্ָণ্ণ & 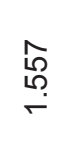 & 음 & 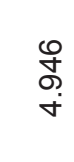 & $\begin{array}{l}\text { Wे } \\
\infty \\
\dot{\forall}\end{array}$ & 0 & $\stackrel{N}{\check{V}}$ & 0 \\
\hline $\begin{array}{l}\frac{\pi}{5} \\
\frac{\sqrt{5}}{\bar{T}} \\
\frac{\pi}{\pi} \\
0\end{array}$ & 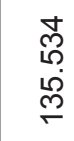 & 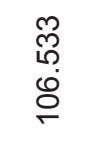 & 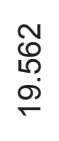 & 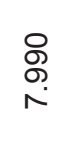 & $\underset{⿱}{\stackrel{g}{+}}$ & 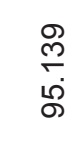 & $\begin{array}{l}\text { ᄋ } \\
\text { م. } \\
\stackrel{1}{1}\end{array}$ & 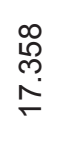 & 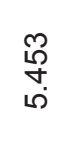 & 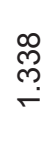 & 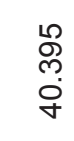 & $\begin{array}{l}\text { W } \\
\text { L़ } \\
\text { مी }\end{array}$ & 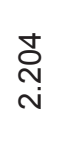 & $\begin{array}{l}\hat{N} \\
\stackrel{\omega}{N} \\
\stackrel{N}{n}\end{array}$ & $\check{F}$ \\
\hline
\end{tabular}




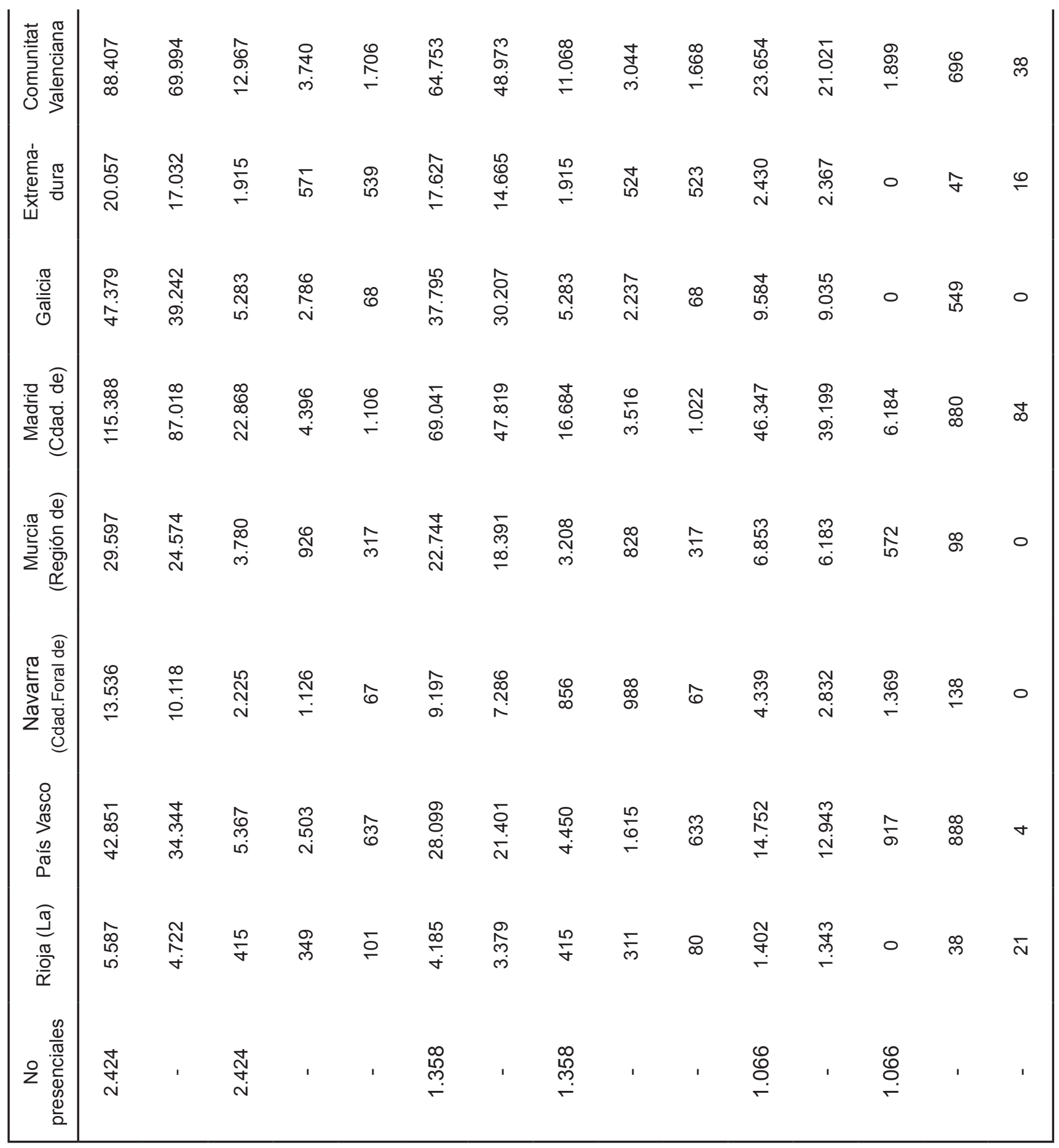

Fuente: Ministerio de Educación, Cultura y Deporte.

No se incluyen Ceuta y Melilla por su escasa representatividad. 
Tabla 5. Distribución porcentual del profesorado según edad por universidad y sexo (2014-15).

\begin{tabular}{|c|c|c|c|c|c|c|c|c|c|c|c|}
\hline & $\begin{array}{l}<30 \\
\text { años }\end{array}$ & $\begin{array}{c}30 \mathrm{a} \\
39\end{array}$ & $\begin{array}{c}40 a \\
49\end{array}$ & $\begin{array}{c}50 a \\
59\end{array}$ & $\begin{array}{l}60 \text { y } \\
\text { más }\end{array}$ & & $\begin{array}{l}<30 \\
\text { años }\end{array}$ & $\begin{array}{c}30 a \\
39\end{array}$ & $\begin{array}{c}40 a \\
49\end{array}$ & $\begin{array}{c}50 a \\
59\end{array}$ & $\begin{array}{l}60 \mathrm{y} \\
\text { más }\end{array}$ \\
\hline $\begin{array}{l}\text { TOTAL } \\
\text { ESPAÑA }\end{array}$ & 2 & 19 & 34 & 32 & 14 & $\begin{array}{c}\text { TOTAL } \\
\text { PRIVADAS }\end{array}$ & 4 & 33 & 34 & 22 & 6 \\
\hline $\begin{array}{c}\text { TOTAL } \\
\text { PÚBLICAS }\end{array}$ & 1 & 16 & 34 & 33 & 15 & $\begin{array}{l}\text { A Distancia de } \\
\text { Madrid }\end{array}$ & 2 & 37 & 40 & 17 & 3 \\
\hline A Coruña & 1 & 18 & 32 & 34 & 14 & Abat Oliba-CEU & 0 & 17 & 40 & 36 & 6 \\
\hline $\begin{array}{l}\text { Alcalá de } \\
\text { Henares }\end{array}$ & 0 & 14 & 33 & 36 & 18 & $\begin{array}{c}\text { Alfonso X El } \\
\text { Sabio }\end{array}$ & 8 & 31 & 38 & 20 & 4 \\
\hline Alicante & 1 & 18 & 41 & 31 & 9 & $\begin{array}{l}\text { Antonio de } \\
\text { Nebrija }\end{array}$ & 3 & 28 & 40 & 22 & 7 \\
\hline Almería & 1 & 12 & 44 & 32 & 11 & $\begin{array}{l}\text { Camilo } \\
\text { José Cela }\end{array}$ & 6 & 33 & 34 & 23 & 5 \\
\hline $\begin{array}{c}\text { Autónoma de } \\
\text { Barcelona }\end{array}$ & 1 & 19 & 32 & 32 & 15 & $\begin{array}{c}\text { Cardenal } \\
\text { Herrera-CEU }\end{array}$ & 9 & 34 & 36 & 18 & 4 \\
\hline $\begin{array}{l}\text { Autónoma de } \\
\text { Madrid }\end{array}$ & 2 & 14 & 30 & 36 & 18 & $\begin{array}{c}\text { Católica de } \\
\text { Valencia }\end{array}$ & 4 & 30 & 33 & 26 & 8 \\
\hline Barcelona & 3 & 18 & 28 & 33 & 18 & $\begin{array}{c}\text { Católica } \\
\text { San Antonio }\end{array}$ & 6 & 44 & 29 & 16 & 5 \\
\hline Burgos & 1 & 16 & 38 & 34 & 10 & $\begin{array}{l}\text { Católica Sta. } \\
\text { T.Jesús Avila }\end{array}$ & 5 & 36 & 39 & 15 & 6 \\
\hline Cádiz & 1 & 15 & 37 & 36 & 10 & Deusto & 3 & 20 & 37 & 30 & 9 \\
\hline Cantabria & 3 & 21 & 28 & 30 & 18 & $\begin{array}{c}\text { Europea de } \\
\text { Canarias }\end{array}$ & 2 & 37 & 48 & 12 & 1 \\
\hline $\begin{array}{c}\text { Carlos III de } \\
\text { Madrid }\end{array}$ & 1 & 28 & 40 & 23 & 7 & $\begin{array}{c}\text { Europea de } \\
\text { Madrid }\end{array}$ & 4 & 51 & 28 & 14 & 3 \\
\hline $\begin{array}{l}\text { Castilla-La } \\
\text { Mancha }\end{array}$ & 1 & 22 & 37 & 31 & 9 & $\begin{array}{c}\text { Europea de } \\
\text { Valencia }\end{array}$ & 7 & 55 & 27 & 9 & 1 \\
\hline $\begin{array}{l}\text { Complutense } \\
\text { de Madrid }\end{array}$ & 0 & 13 & 29 & 35 & 22 & $\begin{array}{c}\text { Europea del } \\
\text { Atlántico }\end{array}$ & 13 & 47 & 19 & 16 & 6 \\
\hline Córdoba & 0 & 16 & 28 & 38 & 17 & $\begin{array}{c}\text { Europea } \\
\text { M.Cervantes }\end{array}$ & 4 & 29 & 46 & 16 & 5 \\
\hline Extremadura & 1 & 15 & 35 & 35 & 14 & $\begin{array}{c}\text { Francisco de } \\
\text { Vitoria }\end{array}$ & 4 & 34 & 37 & 20 & 4 \\
\hline Girona & 5 & 24 & 38 & 27 & 6 & IE Universidad & 2 & 28 & 43 & 21 & 5 \\
\hline Granada & 1 & 14 & 31 & 33 & 20 & $\begin{array}{l}\text { Internacional de } \\
\text { Cataluña }\end{array}$ & 8 & 34 & 29 & 23 & 6 \\
\hline Huelva & 1 & 14 & 46 & 33 & 6 & $\begin{array}{l}\text { Internacional de } \\
\text { la Rioja }\end{array}$ & 4 & 53 & 30 & 12 & 2 \\
\hline Illes Baleares & 3 & 25 & 37 & 25 & 10 & $\begin{array}{l}\text { Internacional } \\
\text { Valenciana }\end{array}$ & 4 & 27 & 54 & 8 & 8 \\
\hline Jaén & 1 & 15 & 48 & 28 & 8 & Isabel I & 11 & 59 & 25 & 5 & 0 \\
\hline
\end{tabular}




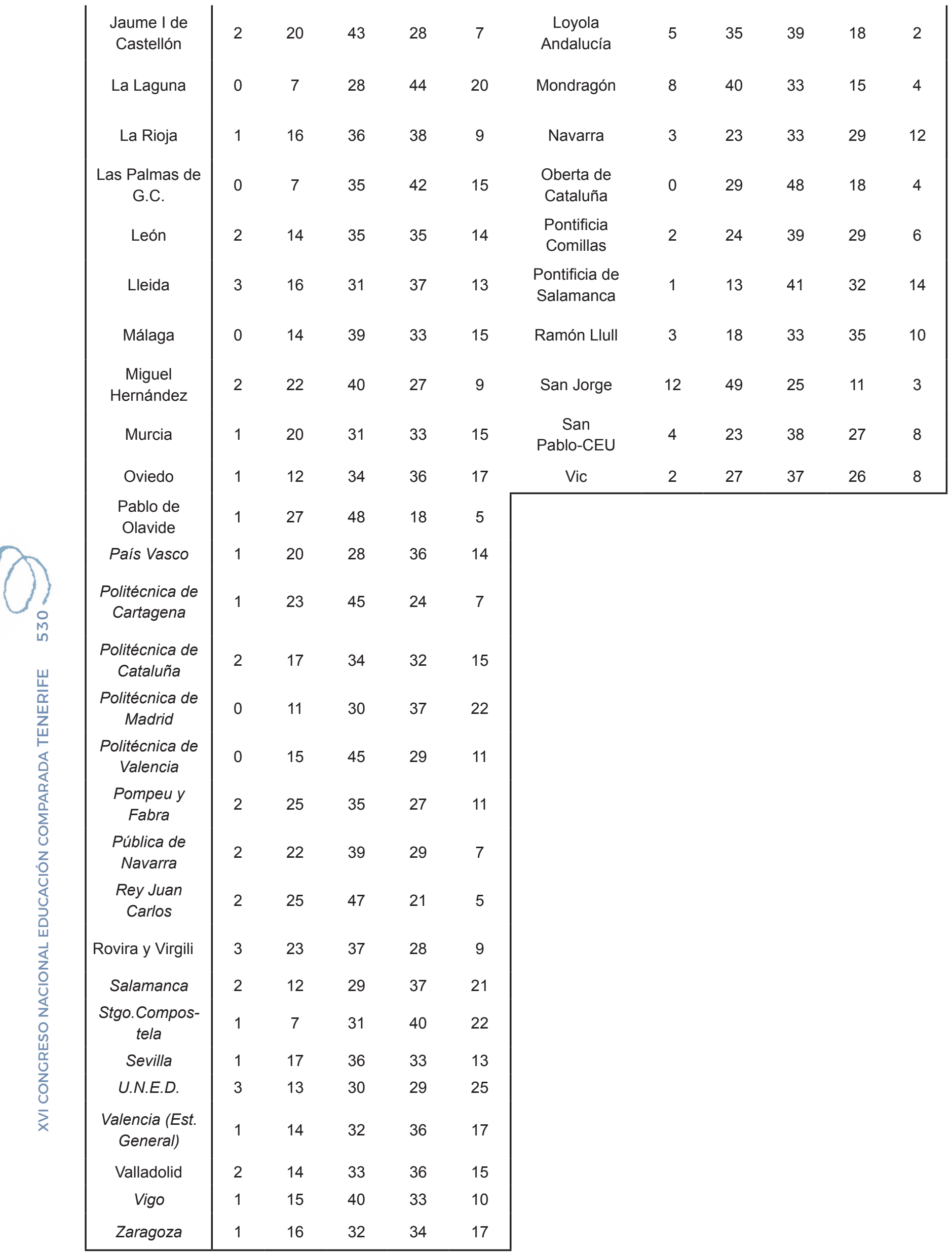

Fuente: Ministerio de Educación, Cultura y Deporte. 
Y también contrasta la mayor juventud del profesorado privado en España, pues un $37 \%$ de los que trabajan en los centros universitarios tienen menos de 40 años, frente a un $17 \%$ de los que lo hacen en universidades públicas (Tabla-5); diferencia más pronunciada en el caso canario, ya que sólo un $7 \%$ de los profesores de ambas universidades públicas tienen menos de 40 años ${ }^{1}$.

La inversión económica es otra variable para analizar los recursos educativos, mostrándose en la Tabla-6 una evolución anual del gasto del conjunto de las Administraciones Públicas inversamente proporcional al esfuerzo realizado por los hogares españoles. El gasto público en 2010 había crecido más de un 34\% en comparación a 2005; y en 2014 ya acumulaba un descenso cercano al 16\% en comparación a 2010; mientras que el dinero gastado por los hogares en educación no ha dejado de crecer, y de un 0,78\% sobre el total del PIB en el año 2008 pasó al 1,11\% en 2014.

a. En Canarias, y pese a que en 2014 su población representaba el 4,6\% del total del país (INE), su participación en el gasto público universitario fue inferior al 3,2\% del total estatal (Tabla-7); dato que debe matizarse:

b. Por políticas de gasto, se observa que el porcentaje de alumnos becados supera el $60 \%$ en la Universidad de La Laguna y roza el $53 \%$ en la de Las Palmas de Gran Canaria, cuando en el conjunto de las universidades públicas españolas es del 39\%. (Tabla-9). De hecho, el gasto público en educación universitaria en Canarias ha descendido, entre 2010 y 2014, dos puntos menos de lo que lo hizo en el conjunto de la nación (Tabla-10).

Y sólo 8 de las 50 universidades públicas españolas tenían en 2015 un endeudamiento financiero inferior al de la Universidad de La Laguna (la Universidad de Las Palmas de Gran Canaria estaba situada en el puesto $17^{\circ}$ entre las de menor endeudamiento); lo que permitía a La Laguna situarse en el puesto $9^{\circ}$ en el ranking de las universidades con mayor independencia financiera (Tabla-11).

Tabla 6. Evolución del gasto público (incluidos capítulos financieros).

\begin{tabular}{|c|c|c|c|c|c|c|c|c|c|c|c|c|c|c|c|c|c|c|c|c|}
\hline & \multicolumn{2}{|c|}{2.005} & \multicolumn{2}{|c|}{2.006} & \multicolumn{2}{|c|}{2.007} & \multicolumn{2}{|c|}{2.008} & \multicolumn{2}{|c|}{2.009} & \multicolumn{2}{|c|}{2.010} & \multicolumn{2}{|c|}{2.011} & \multicolumn{2}{|c|}{2.012} & \multicolumn{2}{|c|}{2.013} & \multicolumn{2}{|c|}{2.014} \\
\hline & 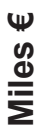 & $\frac{m}{\bar{\alpha}}$ & $\begin{array}{l}\boldsymbol{\Psi} \\
\stackrel{\Phi}{\Sigma} \\
\frac{0}{\Sigma}\end{array}$ & $\begin{array}{l}\frac{m}{\alpha} \\
\therefore\end{array}$ & 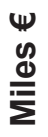 & $\frac{m}{\bar{\alpha}}$ & $\begin{array}{l}\boldsymbol{\Psi} \\
\boldsymbol{\Phi} \\
\stackrel{\underline{\underline{v}}}{\Sigma}\end{array}$ & $\frac{m}{\frac{m}{\alpha}} \frac{0}{\circ}$ & 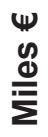 & $\frac{\boldsymbol{m}}{\overline{\mathbf{\alpha}}}$ & $\begin{array}{l}\boldsymbol{\Psi} \\
\boldsymbol{d} \\
\frac{\mathbf{x}}{\Sigma}\end{array}$ & $\frac{m}{\bar{\alpha}}$ & 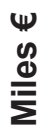 & $\begin{array}{l}\frac{m}{\alpha} \\
\frac{0}{\circ}\end{array}$ & $\begin{array}{l}\boldsymbol{\Psi} \\
\text { d } \\
\frac{0}{\Sigma}\end{array}$ & $\frac{m}{\bar{\alpha}}$ & $\begin{array}{l}\boldsymbol{\Psi} \\
\text { d } \\
\frac{1}{\Sigma}\end{array}$ & $\frac{m}{\bar{\alpha}}$ & $\begin{array}{l}\boldsymbol{\Psi} \\
\underset{\mathcal{O}}{\Sigma} \\
\underline{\Sigma}\end{array}$ & $\frac{m}{\frac{m}{\alpha}}$ \\
\hline 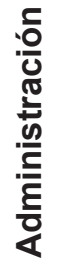 & 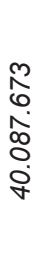 & $\begin{array}{l}n \\
\dot{\sigma}\end{array}$ & $\begin{array}{l}\tilde{m} \\
\text { m} \\
\dot{z} \\
\dot{y} \\
\dot{v}\end{array}$ & $\begin{array}{l}\tilde{m} \\
\nabla^{\prime}\end{array}$ & 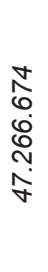 & $\begin{array}{l}\hat{m} \\
\nabla^{\prime}\end{array}$ & $\begin{array}{l}\infty \\
8 \\
0 \\
\frac{1}{N} \\
\frac{1}{n}\end{array}$ & 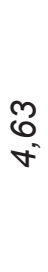 & 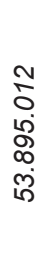 & $\begin{array}{l}\text { S } \\
\forall\end{array}$ & 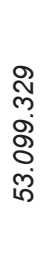 & $\begin{array}{l}5 \\
\sigma\end{array}$ & $\begin{array}{l}\infty \\
0 \\
0 \\
\bar{ల} \\
6 \\
0 \\
0\end{array}$ & $\stackrel{\Re}{\curvearrowright}$ & 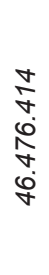 & $\begin{array}{l}\hat{y} \\
\forall\end{array}$ & 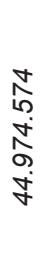 & $\begin{array}{l}\text { ఇ } \\
\forall\end{array}$ & 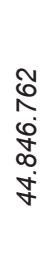 & 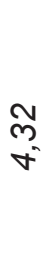 \\
\hline 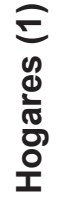 & $\begin{array}{l}\mathbb{1} \\
\infty \\
\infty \\
\infty\end{array}$ & $\begin{array}{l}\hat{\infty} \\
0^{\prime \prime}\end{array}$ & $\begin{array}{l}\bar{y} \\
\infty \\
\infty\end{array}$ & $\begin{array}{l}\forall \\
\infty \\
0 \\
0\end{array}$ & 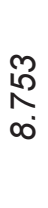 & $\begin{array}{l}\overline{1} \\
\infty \\
0\end{array}$ & $\begin{array}{l}\stackrel{\curvearrowright}{N} \\
\infty\end{array}$ & $\frac{\infty}{0}$ & $\begin{array}{l}n \\
0 \\
0\end{array}$ & $\begin{array}{l}\text { వ } \\
\infty \\
0\end{array}$ & $\begin{array}{l}\infty \\
\text { లి } \\
\text { ஸे }\end{array}$ & $\begin{array}{l}0 \\
\infty \\
0\end{array}$ & $\begin{array}{l}\stackrel{2}{\hat{N}} \\
\sigma^{\circ}\end{array}$ & $\begin{array}{l}\tilde{\sigma} \\
0\end{array}$ & 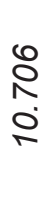 & $\stackrel{m}{a}$ & $\begin{array}{l}\stackrel{0}{N} \\
\stackrel{m}{r}\end{array}$ & $\approx$ & $\begin{array}{l}8 \\
6 \\
2 \\
2\end{array}$ & $\stackrel{F}{2}$ \\
\hline
\end{tabular}

Fuente: Ministerio de Educación, Cultura y Deporte.

(1) Pagos de las familias a centros y clases particulares (no incluye servicios complementarios ni en bienes educativos).

\footnotetext{
${ }^{1}$ Esta situación no se da en la única universidad privada que existía en las islas en el curso 2014/15, ya que el 39\% de sus profesores tenían menos de 40 años (Tabla-5).
} 
Tabla 7. \% Participación en el gasto público en educación universitaria (2014).

\begin{tabular}{|c|c|}
\hline Total España (miles $€$ ) & 8.889 .702 \\
\hline Ministerio Educación & 2,7 \\
\hline Andalucía & 18,9 \\
\hline Aragón & 2,8 \\
\hline Asturias (Ppdo. de) & 2,0 \\
\hline Balears (IIles) & 1,0 \\
\hline Canarias & 3,2 \\
\hline Cantabria & 1,2 \\
\hline Castilla y León & 5,6 \\
\hline Castilla-La Mancha & 2,1 \\
\hline Cataluña & 15,2 \\
\hline C.Valenciana & 12,5 \\
\hline Extremadura & 1,7 \\
\hline Galicia & 5,8 \\
\hline Madrid (Cdad. de) & 16,4 \\
\hline Murcia (Región de) & 2,8 \\
\hline Navarra (Cdad. Foral de) & 0,8 \\
\hline País Vasco & 4,9 \\
\hline Rioja (La) & 0,5 \\
\hline No Educativas (2) & 0,2 \\
\hline
\end{tabular}

Fuente: Ministerio de Educación, Cultura y Deporte.

(2) Incluye otros Ministerios, Consejerías y CCLL. No se incluye Ceuta y Melilla.
Tabla 8. \% Gasto público de cada Comunidad destinado a Educación Universitaria.

\begin{tabular}{|c|c|}
\hline Total España & 20,9 \\
\hline Ministerio Educación & 11,9 \\
\hline Andalucía & 23,1 \\
\hline Aragón & 24,3 \\
\hline Asturias (Ppdo. de) & 22,8 \\
\hline Balears (IIles) & 11,5 \\
\hline Canarias & 18,4 \\
\hline Cantabria & 19,3 \\
\hline Castilla y León & 24,6 \\
\hline Castilla-La Mancha & 12,5 \\
\hline Cataluña & 24,3 \\
\hline $\begin{array}{l}\text { Comunitat Valencia- } \\
\text { na }\end{array}$ & 26,6 \\
\hline Extremadura & 15,3 \\
\hline Galicia & 23,4 \\
\hline Madrid (Cdad de) & 31,3 \\
\hline Murcia (Región de) & 19,8 \\
\hline $\begin{array}{c}\text { Navarra (Cdad. Foral } \\
\text { de) }\end{array}$ & 12,8 \\
\hline País Vasco & 16,9 \\
\hline Rioja (La) & 15,8 \\
\hline No Educativas (2) & 0,4 \\
\hline
\end{tabular}


Tabla 9. Becarios y becas en enseñanza universitaria.

\begin{tabular}{|c|c|c|c|c|c|c|c|}
\hline & 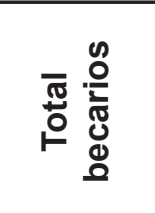 & 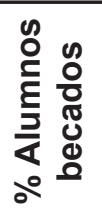 & 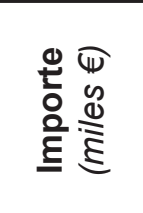 & & 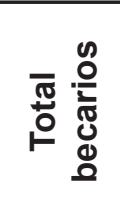 & 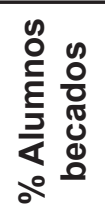 & 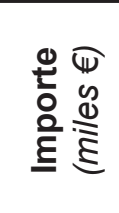 \\
\hline TOTAL & 524.589 & 34,8 & 964.654 & Universidades privadas & 20.409 & 9,2 & 39.247 \\
\hline Universidades públicas & 504.175 & 39,2 & 925.404 & A Distancia de Madrid & 262 & 3,7 & 430 \\
\hline A Coruña & 6.287 & 34,6 & 12.861 & Abat Oliba CEU & 25 & 1,4 & 59 \\
\hline Alcalá & 6.523 & 38,0 & 12.704 & Alfonso X El Sabio & 206 & 2,8 & 597 \\
\hline Alicante & 20.871 & 80,2 & 26.824 & Antonio de Nebrija & 196 & 4,5 & 551 \\
\hline Almería & 5.483 & 43,6 & 12.436 & Camilo José Cela & 418 & 4,5 & 1.098 \\
\hline Autónoma de Barcelona & 13.442 & 36,5 & 26.056 & Cardenal Herrera-CEU & 1.534 & 21,7 & 2.176 \\
\hline Autónoma de Madrid & 10.699 & 40,9 & 19.849 & Católica de Valencia & 4.368 & 39,4 & 6.308 \\
\hline Barcelona & 17.953 & 35,3 & 32.602 & Católica San Antonio & 1.464 & 12,6 & 3.614 \\
\hline Burgos & 3.058 & 40,1 & 5.431 & Católica S.T.Jesús Ávila & 146 & 5,4 & 354 \\
\hline Cádiz & 10.355 & 50,4 & 21.221 & Deusto & 1.681 & 20,1 & 2.681 \\
\hline Cantabria & 4.294 & 40,8 & 7.361 & Europea de Canarias & 25 & 10,0 & 67 \\
\hline Carlos III de Madrid & 6.799 & 38,2 & 11.220 & Europea de Madrid & 415 & 3,3 & 1.201 \\
\hline Castilla-La Mancha & 11.947 & 46,2 & 25.572 & Europea de Valencia & 24 & 3,7 & 26 \\
\hline Complutense de Madrid & 25.836 & 38,0 & 46.091 & Europea del Atlántico & 97 & 28,4 & 142 \\
\hline Córdoba & 8.094 & 43,2 & 17.873 & Europea M.Cervantes & 157 & 14,9 & 330 \\
\hline Extremadura & 11.349 & 54,0 & 28.472 & Francisco de Vitoria & 360 & 5,6 & 931 \\
\hline Girona & 4.964 & 34,1 & 9.960 & IE Universidad & 11 & 0,3 & 44 \\
\hline Granada & 20.326 & 38,7 & 53.974 & Intern.Catalunya & 235 & 7,0 & 593 \\
\hline Huelva & 4.985 & 44,4 & 11.055 & Intern.e La Rioja & 799 & 4,7 & 1.072 \\
\hline Illes Balears & 4.420 & 32,8 & 7.203 & Intern.Valenciana & 465 & 21,1 & 527 \\
\hline Intern.Andalucía & 31 & 15,3 & 101 & Isabel I & 171 & 5,3 & 302 \\
\hline Int.Menéndez Pelayo & 132 & 12,0 & 296 & Loyola Andalucia & 120 & 9,1 & 263 \\
\hline Jaén & 7.208 & 48,1 & 16.228 & Mondragon Unib. & 846 & 20,6 & 1.480 \\
\hline Jaume I de Castellón & 8.677 & 64,6 & 10.949 & Navarra & 1.463 & 15,2 & 4.099 \\
\hline
\end{tabular}




\begin{tabular}{|c|c|c|c|c|c|c|c|}
\hline La Laguna & 12.384 & 60,2 & 24.594 & Oberta de Catalunya & 1.193 & 3,0 & 1.173 \\
\hline La Rioja & 1.579 & 36,9 & 3.069 & Pontificia Comillas & 702 & 8,9 & 1.784 \\
\hline Las Palmas de G.C. & 10.241 & 52,9 & 18.729 & Pontificia de Salamanca & 714 & 14,2 & 1.822 \\
\hline León & 4.705 & 34,7 & 10.106 & Ramon Llull & 741 & 4,7 & 1.475 \\
\hline Lleida & 3.463 & 38,3 & 7.270 & San Jorge & 178 & 8,6 & 408 \\
\hline Málaga & 17.262 & 46,5 & 37.681 & San Pablo CEU & 351 & 3,6 & 1.063 \\
\hline Miguel Hernández & 7.986 & 59,9 & 10.948 & Vic & 1.042 & 18,4 & 2.577 \\
\hline Murcia & 14.109 & 46,1 & 28.186 & Sin especificar & 5 & - & 2 \\
\hline Oviedo & 7.322 & 33,5 & 14.791 & & & & \\
\hline Pablo de Olavide & 4.928 & 48,4 & 10.728 & & & & \\
\hline País Vasco & 13.567 & 32,2 & 23.794 & & & & \\
\hline Politécnica de Cartagena & 1.816 & 31,8 & 3.023 & & & & \\
\hline Politécnica de Catalunya & 8.811 & 28,6 & 14.225 & & & & \\
\hline Politécnica de Madrid & 10.382 & 29,4 & 15.366 & & & & \\
\hline Politécnica de Valencia & 17.827 & 58,8 & 24.473 & & & & \\
\hline Pompeu Fabra & 5.375 & 35,0 & 9.673 & & & & \\
\hline Pública de Navarra & 3.485 & 44,6 & 5.116 & & & & \\
\hline Rey Juan Carlos & 14.095 & 36,5 & 24.767 & & & & \\
\hline Rovira i Virgili & 5.495 & 42,7 & 11.296 & & & & \\
\hline Salamanca & 11.100 & 45,6 & 26.177 & & & & \\
\hline Santiago de Compostela & 8.762 & 36,9 & 22.221 & & & & \\
\hline Sevilla & 26.501 & 43,4 & 50.257 & & & & \\
\hline UNED & 17.244 & 10,9 & 12.391 & & & & \\
\hline Valencia Estudi General & 33.844 & 73,3 & 46.983 & & & & \\
\hline Valladolid & 9.913 & 41,4 & 18.666 & & & & \\
\hline Vigo & 8.153 & 43,2 & 16.066 & & & & \\
\hline Zaragoza & 10.093 & 33,3 & 18.468 & & & & \\
\hline
\end{tabular}

Fuente: Ministerio de Educación, Cultura y Deporte. 
Tabla 10. Evolución del gasto público en educación universitaria (miles de $€$ ).

\begin{tabular}{|c|c|c|c|c|c|c|c|c|c|}
\hline & $\stackrel{10}{\stackrel{2}{2}}$ & $\stackrel{\nabla}{\stackrel{N}{N}}$ & $\stackrel{m}{\stackrel{m}{N}}$ & $\stackrel{N}{\text { ㅇ }}$ & $\bar{\delta}$ & 웅 & ஜ̊ & ঃి & $\stackrel{\circ}{\circ}$ \\
\hline$\frac{\frac{\pi}{0}}{\frac{0}{\pi}}$ & 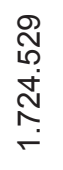 & $\begin{array}{l}\overleftarrow{\sigma} \\
\dot{\sigma} \\
\stackrel{R}{0} \\
\dot{\sigma}\end{array}$ & $\begin{array}{l}\stackrel{m}{o} \\
\stackrel{+}{+} \\
\dot{\infty} \\
\stackrel{0}{+}\end{array}$ & $\begin{array}{l}\stackrel{0}{0} \\
\infty \\
\stackrel{N}{N} \\
\stackrel{N}{r}\end{array}$ & $\begin{array}{l}\text { J } \\
\infty \\
0 \\
0 \\
\infty \\
\infty \\
-\end{array}$ & 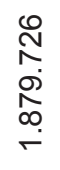 & 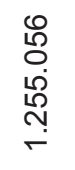 & $\begin{array}{l}\stackrel{0}{\infty} \\
\stackrel{+}{*} \\
\infty\end{array}$ & $\begin{array}{l}\stackrel{0}{0} \\
\stackrel{N}{N} \\
\hat{\sigma}\end{array}$ \\
\hline 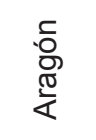 & 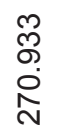 & $\stackrel{\bar{n}}{\stackrel{5}{5}}$ & 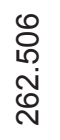 & 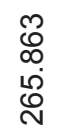 & 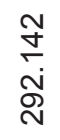 & $\begin{array}{l}\stackrel{\circ}{2} \\
\stackrel{N}{0} \\
\stackrel{0}{े}\end{array}$ & 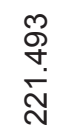 & 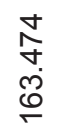 & $\begin{array}{l}\hat{N} \\
\stackrel{+}{+} \\
\stackrel{+}{+}\end{array}$ \\
\hline 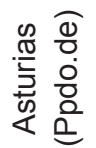 & $\begin{array}{l}\infty \\
\infty \\
\infty \\
\overleftarrow{\infty} \\
\leftarrow\end{array}$ & $\begin{array}{l}\stackrel{g}{+} \\
\stackrel{i}{0} \\
\stackrel{0}{\sigma}\end{array}$ & 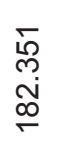 & 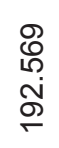 & 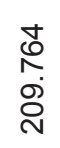 & $\begin{array}{l}\bar{\infty} \\
\infty \\
\stackrel{\infty}{0} \\
\stackrel{N}{N}\end{array}$ & 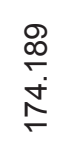 & 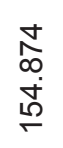 & $\begin{array}{l}\stackrel{\infty}{\stackrel{2}{*}} \\
\stackrel{\infty}{\leftarrow}\end{array}$ \\
\hline 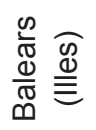 & 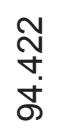 & $\begin{array}{l}\text { ఫे } \\
\text { ల. } \\
\infty \\
\infty\end{array}$ & $\begin{array}{l}\text { Dొ } \\
\stackrel{0}{0} \\
\infty \\
\infty\end{array}$ & 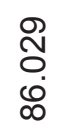 & $\frac{5}{\frac{5}{5}}$ & $\begin{array}{l}\text { ô } \\
\text { ஸे }\end{array}$ & 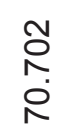 & $\begin{array}{l}\infty \\
\stackrel{\infty}{m} \\
\stackrel{\sigma}{\sigma}\end{array}$ & ' \\
\hline 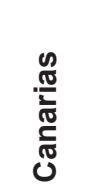 & 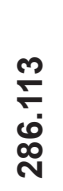 & 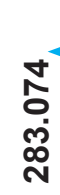 & 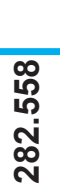 & 品 & $\begin{array}{l}\frac{T}{\hat{\sigma}} \\
\stackrel{m}{m}\end{array}$ & $\begin{array}{l}\text { ơ } \\
\stackrel{\infty}{0} \\
\text { స్ల }\end{array}$ & 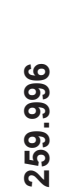 & 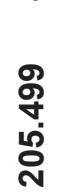 & $\begin{array}{l}\text { ̃े } \\
\infty \\
\dot{0} \\
\dot{0}\end{array}$ \\
\hline $\begin{array}{l}\frac{\pi}{\frac{\pi}{2}} \\
\frac{\pi}{0} \\
\frac{\pi}{\pi} \\
0 \\
0\end{array}$ & $\begin{array}{l}\infty \\
8 \\
\stackrel{0}{\circ}\end{array}$ & $\begin{array}{l}\text { ల్ } \\
\text { ळ. } \\
\stackrel{\sigma}{\sigma}\end{array}$ & \begin{tabular}{l}
0 \\
$\infty$ \\
$\infty$ \\
1 \\
0 \\
\hdashline
\end{tabular} & $\begin{array}{l}\stackrel{m}{\Lambda} \\
\infty \\
\dot{\sim} \\
\stackrel{5}{\leftarrow}\end{array}$ & 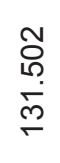 & 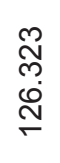 & $\begin{array}{l}\text { J } \\
\text { D. } \\
\infty \\
\infty\end{array}$ & $\begin{array}{l}\stackrel{0}{N} \\
\stackrel{R}{R}\end{array}$ & 을 \\
\hline 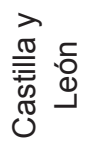 & $\begin{array}{l}\stackrel{N}{\infty} \\
\stackrel{\infty}{\oplus} \\
\stackrel{\circ}{\circ}\end{array}$ & 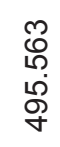 & $\begin{array}{l}\bar{S} \\
\text { } \\
\text { O্ণ }\end{array}$ & $\begin{array}{l}\stackrel{m}{0} \\
\stackrel{0}{0} \\
\infty \\
\rho \\
P\end{array}$ & 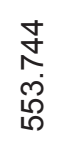 & 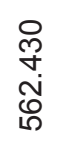 & $\begin{array}{l}0 \\
\tilde{ల} \\
\dot{\tilde{D}} \\
\tilde{ల}\end{array}$ & 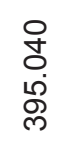 & $\begin{array}{l}\stackrel{+}{్} \\
\stackrel{N}{N}\end{array}$ \\
\hline 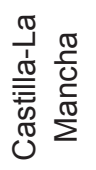 & 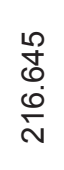 & $\begin{array}{l}\hat{N} \\
\stackrel{N}{\infty} \\
\stackrel{\infty}{\infty}\end{array}$ & $\begin{array}{l}\bar{\sigma} \\
\dot{\infty} \\
\leftarrow\end{array}$ & 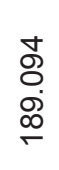 & $\begin{array}{l}\stackrel{m}{\sigma} \\
\stackrel{0}{0} \\
\stackrel{\oplus}{N}\end{array}$ & 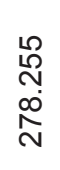 & $\begin{array}{l}\infty \\
\tilde{O} \\
\stackrel{0}{\rho} \\
\stackrel{\rho}{\sigma}\end{array}$ & 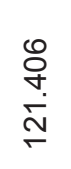 & $\begin{array}{l}\text { P } \\
\stackrel{m}{0} \\
\dot{0}\end{array}$ \\
\hline $\begin{array}{l}\frac{\pi}{5} \\
\frac{\pi}{5} \\
\frac{\pi}{\pi} \\
0\end{array}$ & $\begin{array}{l}\underset{+}{+} \\
\stackrel{+}{+} \\
\stackrel{+}{+}\end{array}$ & 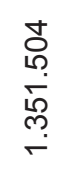 & $\begin{array}{l}8 \\
0 \\
+ \\
o \\
o \\
\stackrel{+}{+}\end{array}$ & 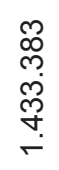 & 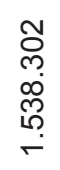 & 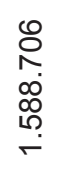 & 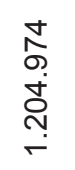 & $\begin{array}{l}\text { ષ } \\
\infty \\
\mathbb{N} \\
\infty\end{array}$ & \begin{tabular}{l}
$\overline{0}$ \\
ల. \\
\multirow{J}{*}{}
\end{tabular} \\
\hline 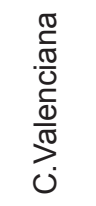 & 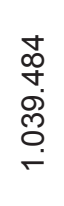 & 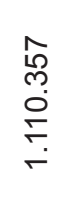 & 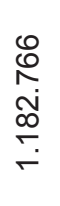 & 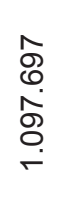 & $\begin{array}{l}\infty \\
0 \\
\infty \\
\infty \\
\infty \\
\infty \\
\check{\Gamma}\end{array}$ & 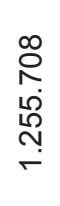 & $\begin{array}{l}\mathscr{\&} \\
\mathbb{O} \\
\stackrel{\infty}{\mathcal{D}} \\
\infty\end{array}$ & $\begin{array}{l}\text { N్ } \\
\text { స్ } \\
\widetilde{0}\end{array}$ & \begin{tabular}{l}
$\hat{Q}$ \\
$\stackrel{N}{ }$ \\
$\infty$ \\
\multirow{\gamma}{*}{}
\end{tabular} \\
\hline 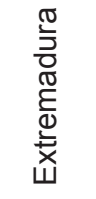 & 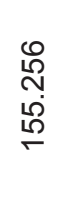 & $\begin{array}{l}\mathscr{O} \\
\infty \\
\infty \\
\dot{f}\end{array}$ & 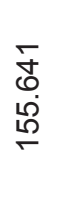 & \begin{tabular}{l}
$\stackrel{N}{N}$ \\
N \\
\multirow{T}{*}{}
\end{tabular} & 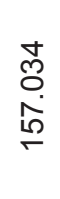 & $\frac{N}{N}$ & $\begin{array}{l}\hat{N} \\
\hat{\omega} \\
\stackrel{N}{F}\end{array}$ & $\begin{array}{l}\text { ட̊ } \\
\text { ○. } \\
\mathscr{\theta}\end{array}$ & $\begin{array}{l}\text { ఠ్ } \\
0 \\
\dot{\sigma}\end{array}$ \\
\hline $\begin{array}{l}\frac{\pi}{0.0} \\
\frac{0}{\pi} \\
0 \\
0\end{array}$ & 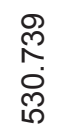 & 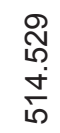 & \begin{tabular}{l}
$N$ \\
\multirow{5}{*}{} \\
$\stackrel{5}{5}$ \\
$i n$
\end{tabular} & 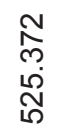 & 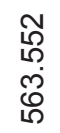 & $\begin{array}{l}\stackrel{8}{0} \\
\text { ల) } \\
\text { ర్ర }\end{array}$ & $\begin{array}{l}\hat{R} \\
\dot{0} \\
\stackrel{0}{\circ}\end{array}$ & 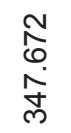 & $\begin{array}{l}0 \\
\infty \\
\infty \\
\\
\stackrel{N}{N}\end{array}$ \\
\hline
\end{tabular}




\begin{tabular}{|c|c|c|c|c|c|c|c|c|c|c|}
\hline 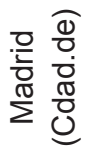 & $\begin{array}{l}\infty \\
\infty \\
0 \\
\dot{-} \\
\infty \\
+ \\
+ \\
\dot{-}\end{array}$ & 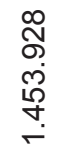 & 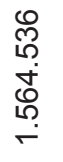 & 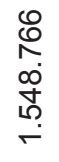 & & $\begin{array}{l}\frac{5}{n} \\
\text { m. } \\
\stackrel{0}{\rho} \\
\stackrel{-}{r}\end{array}$ & $\begin{array}{l}\hat{0} \\
\stackrel{0}{10} \\
\infty \\
\stackrel{0}{r}\end{array}$ & 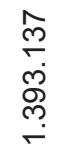 & $\begin{array}{l}\bar{\Lambda} \\
0 \\
0 \\
b \\
\infty\end{array}$ & $\begin{array}{l}\infty \\
\infty \\
\infty \\
\infty \\
0 \\
0\end{array}$ \\
\hline 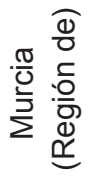 & $\begin{array}{l}\bar{\sigma} \\
\infty \\
\infty \\
\stackrel{d}{d} \\
\text { d }\end{array}$ & 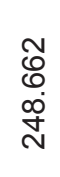 & 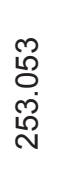 & 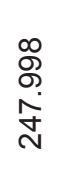 & & 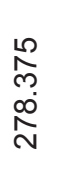 & 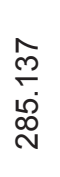 & $\begin{array}{l}\stackrel{\circ}{m} \\
\stackrel{\circ}{N}\end{array}$ & 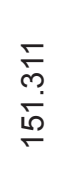 & $\begin{array}{l}\infty \\
\stackrel{m}{i} \\
\infty \\
\infty\end{array}$ \\
\hline 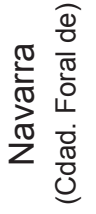 & 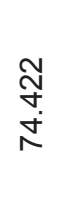 & \begin{tabular}{l}
8 \\
$\infty$ \\
\multirow{N}{+}{}
\end{tabular} & $\begin{array}{l}\text { Uి } \\
\dot{N} \\
\text { I }\end{array}$ & 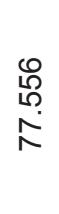 & & $\begin{array}{l}\stackrel{\infty}{\circ} \\
\infty \\
\infty \\
\infty \\
\infty\end{array}$ & $\begin{array}{l}0 \\
0 \\
10 \\
\infty \\
\infty\end{array}$ & \begin{tabular}{l}
\multirow{+}{*}{} \\
$\infty$ \\
0 \\
0
\end{tabular} & 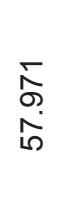 & $\begin{array}{l}\frac{10}{0} \\
\dot{0} \\
\dot{e}\end{array}$ \\
\hline 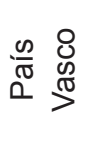 & $\begin{array}{l}\stackrel{\infty}{N} \\
\text { } \\
\text { ஸे } \\
\stackrel{+}{+}\end{array}$ & 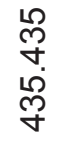 & 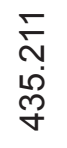 & 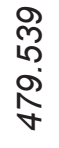 & & $\begin{array}{l}\underset{N}{N} \\
\text { ஜ் } \\
\stackrel{0}{0}\end{array}$ & $\frac{\infty}{\stackrel{\infty}{\circ}}$ & $\begin{array}{l}\stackrel{0}{\circ} \\
\stackrel{\leftrightarrow}{R} \\
\dot{J} \\
\text { J }\end{array}$ & $\begin{array}{l}\text { N } \\
\stackrel{N}{N} \\
\stackrel{N}{N}\end{array}$ & $\begin{array}{l}\stackrel{0}{0} \\
\infty \\
\infty \\
\infty \\
\infty\end{array}$ \\
\hline $\begin{array}{l}\frac{\sqrt[\sigma]{\sigma}}{\frac{\sigma}{\sigma}} \\
\widetilde{\sigma}\end{array}$ & $\begin{array}{l}\infty \\
\stackrel{0}{0} \\
\infty \\
\stackrel{0}{+}\end{array}$ & 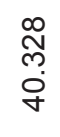 & $\frac{\stackrel{N}{N}}{\dot{+}}$ & $\begin{array}{c}\bar{m} \\
\dot{\gamma} \\
\dot{\gamma}\end{array}$ & & 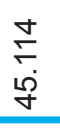 & $\begin{array}{l}\mathscr{8} \\
0 \\
\dot{\varphi}\end{array}$ & $\begin{array}{l}8 \\
\stackrel{8}{1} \\
\text { ip }\end{array}$ & $\begin{array}{l}\text { ర్ల } \\
\text { } \\
\text { లె }\end{array}$ & 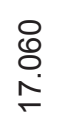 \\
\hline 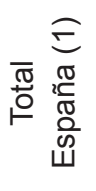 & 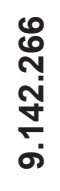 & 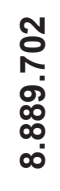 & 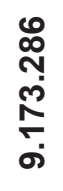 & $\begin{array}{l}0 \\
\text { 우 } \\
\text { ஸें } \\
\text { ò }\end{array}$ & & 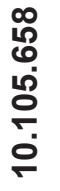 & 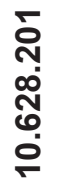 & 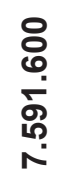 & 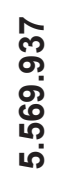 & 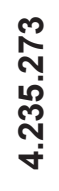 \\
\hline $\begin{array}{l}\widehat{\mathbb{N}} \\
\mathscr{D} \\
\mathbb{J} \\
\mathbb{D} \\
\infty\end{array}$ & $\begin{array}{l}\infty \\
\stackrel{0}{*} \\
\infty \\
1 \\
0\end{array}$ & 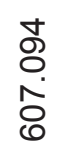 & $\underset{\substack{N \\
\stackrel{n}{n}}}{\stackrel{n}{n}}$ & $\begin{array}{l}\stackrel{m}{+} \\
\stackrel{N}{N} \\
\stackrel{N}{N}\end{array}$ & & 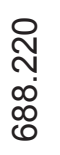 & 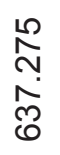 & $\begin{array}{l}\frac{1}{10} \\
10 \\
10 \\
10 \\
+\end{array}$ & 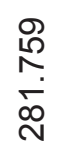 & $\begin{array}{l}\bar{m} \\
\text { م్ } \\
\text { ల్ల } \\
\end{array}$ \\
\hline
\end{tabular}

Fuente: M. ${ }^{\circ}$ Educación, Cultura y Deporte (Cuestionario UOE).

(1): Incluye Comunidades Autónomas, Gobierno de España y Universidades públicas.

(2): Excepto exensión de precios académicos. 
Tabla 11. Indicadores financieros de las universidades públicas 2015.

\begin{tabular}{|c|c|c|}
\hline$(\%)$ & Endeudamiento & $\begin{array}{c}\text { Independencia financie- } \\
\text { ra }\end{array}$ \\
\hline A Coruña & 19,18 & 80,82 \\
\hline Alcalá & 13,93 & 86,07 \\
\hline Alicante & 32,52 & 67,48 \\
\hline Almería & 11,5 & 88,5 \\
\hline Autónoma de Barcelona & 41,78 & 58,22 \\
\hline Autónoma de Madrid & 58,46 & 41,54 \\
\hline Barcelona & 48,25 & 51,75 \\
\hline Burgos & 16,61 & 83,39 \\
\hline Cádiz & 7,66 & 92,34 \\
\hline Cantabria & 29,11 & 70,89 \\
\hline Carlos III de Madrid & 31,44 & 68,56 \\
\hline Castilla-La Mancha & 16,4 & 83,6 \\
\hline Complutense de Madrid & 20,63 & 79,37 \\
\hline Córdoba & 6,67 & 93,33 \\
\hline Extremadura & 22,3 & 77,7 \\
\hline Girona & 29,11 & 70,89 \\
\hline Granada & 20,17 & 79,83 \\
\hline Huelva & 25,67 & 74,33 \\
\hline Illes Balears (Les) & 38,39 & 61,61 \\
\hline Internacional de Andalucía & 22,25 & 77,75 \\
\hline I.Menéndez Pelayo & 13,05 & 86,95 \\
\hline Jaén & 9,86 & 90,14 \\
\hline Jaume I de Castellón & 26,28 & 73,72 \\
\hline La Laguna & 13,44 & 86,56 \\
\hline La Rioja & 31,19 & 68,81 \\
\hline Las Palmas de Gran Canaria & 18,68 & 81,32 \\
\hline León & 19,33 & 80,67 \\
\hline
\end{tabular}




\begin{tabular}{|c|c|c|}
\hline Lleida & 18,83 & 81,17 \\
\hline Málaga & 15,87 & 84,13 \\
\hline Miguel Hernández de Elche & 13,82 & 86,18 \\
\hline Murcia & 25,08 & 74,92 \\
\hline UNED & 53,71 & 46,29 \\
\hline Oviedo & 18,8 & 81,2 \\
\hline Pablo de Olavide & 14,22 & 85,78 \\
\hline País Vasco/Euskal H.U. & 43,64 & 56,36 \\
\hline Politécnica de Cartagena & 22,38 & 77,62 \\
\hline Politécnica de Catalunya & 66,83 & 33,17 \\
\hline Politécnica de Madrid & 40,78 & 59,22 \\
\hline Politècnica de València & 24,76 & 75,24 \\
\hline Pompeu Fabra & 41,97 & 58,03 \\
\hline Pública de Navarra & 5,49 & 94,51 \\
\hline Rey Juan Carlos & 23,01 & 76,99 \\
\hline Rovira i Virgili & 30,45 & 69,55 \\
\hline Salamanca & 20,05 & 79,95 \\
\hline Santiago de Compostela & 26,89 & 73,11 \\
\hline Sevilla & 10 & 90 \\
\hline València (Estudi General) & 23,53 & 76,47 \\
\hline Valladolid & 15,27 & 84,73 \\
\hline Vigo & 12,81 & 87,19 \\
\hline Zaragoza & 19,39 & 80,61 \\
\hline
\end{tabular}

Fuente: INE (M. ${ }^{\circ}$ Educación, Cultura y Deporte). 
- II. La escolarización. Las transiciones del sistema educativo universitario se resumen con cuatro indicadores agrupados en dos bloques ilustrativos de su evolución temporal y de la perspectiva comparada autonómica:

1. La tasa neta de escolarización universitaria en Canarias entre los 18 y 24 años es inferior al conjunto de España en cada una de las edades (Tabla-12). Por ejemplo, con 21 años, sólo en Baleares (12,6\%) y Castilla-La Mancha (20\%), hay menos universitarios que en Canarias $(23,4 \%)$, estando situada la tasa media española de dicha edad en el $37,8 \%$. Aunque parezca elevada la cifra de 6.550 personas que finalizaron en 2014 algún tipo de estudio superior en Canarias (Tabla-14), sólo el otro archipiélago español tuvo en el curso 2013/14 una tasa bruta de población que finalizó un Grado inferior (Tabla-13).

2. La población entre 30 y 34 años con estudios superiores está descendiendo lentamente en Canarias, pues de un 33,6\% en 2005 se pasó a un 33,1\% en 2010, y a un 32,9\% en 2015 (Tabla-15). Este descenso de 0,2 puntos en el último lustro es inferior al experimentado por la media española para dicha edad (-1,1 puntos), pero en 2015 el porcentaje de españoles entre 30 y 34 años con estudios superiores era del $41 \%$. Es decir, Canarias estaba situada 8 puntos por debajo del conjunto del Estado pese a experimentar un descenso más suave en los dos últimos lustros.

- III. Los procesos educativos. Dos de los aspectos más relevantes de los procesos educativos universitarios se refieren al éxito en el acceso a la misma y la tipología de la relación laboral de los profesores. Para analizar el primero, se han eliminado los sistemas para mayores de 25 y 45 años, así como para mayores de 40 por experiencia profesional acreditada, ya que dificultan concretar el éxito desde los itinerarios preuniversitarios. Es muy destacado el aumento que ha experimentado Canarias en la tasa de bruta de población que supera el acceso a la universidad (Tabla-16), que de un 33,1 en 2005 se incrementó hasta el 38,5 en 2010, y en 2015 prácticamente alcanzó la media nacional, situándose en el 50,2 cuando la media del Estado era del 51,8. 
Tabla 12. Tasas netas de escolarización universitaria.

\begin{tabular}{|c|c|c|c|c|c|c|c|}
\hline & 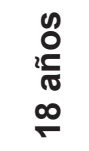 & 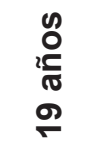 & 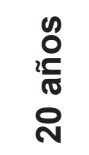 & $\begin{array}{l}\stackrel{0}{\circ} \\
\frac{2}{\pi} \\
\grave{N}\end{array}$ & 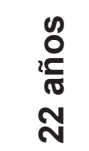 & 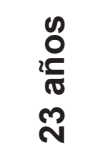 & 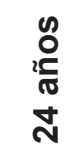 \\
\hline$\frac{1}{0}$ & 31,6 & 35,5 & 37,3 & 37,8 & 31,7 & 25,9 & 19,3 \\
\hline$\frac{\frac{\pi}{0}}{\frac{\pi}{\pi}}$ & 27,5 & 31,5 & 33,7 & 33,8 & 27,8 & 23,6 & 17,7 \\
\hline $\begin{array}{l}\stackrel{5}{\delta} \\
\stackrel{\mathbb{\pi}}{\tau}\end{array}$ & 34,1 & 39,9 & 40,7 & 39,4 & 30,1 & 24,3 & 16,3 \\
\hline 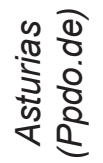 & 37,0 & 38,3 & 37,2 & 36,0 & 29,7 & 22,5 & 17,7 \\
\hline 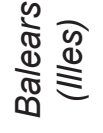 & 11,9 & 14,6 & 15,0 & 15,5 & 12,6 & 10,5 & 8,3 \\
\hline 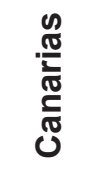 & 21,7 & 23,4 & 23,6 & 23,4 & 18,0 & 15,0 & 11,0 \\
\hline 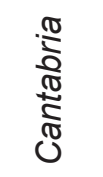 & 27,3 & 30,0 & 30,7 & 30,9 & 25,6 & 21,5 & 16,5 \\
\hline 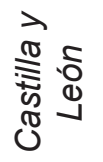 & 38,0 & 44,7 & 46,5 & 46,5 & 37,7 & 31,4 & 23,8 \\
\hline 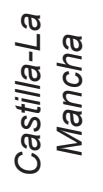 & 14,1 & 16,4 & 17,8 & 20,0 & 16,2 & 13,4 & 9,2 \\
\hline$\frac{\mathbb{N}}{\stackrel{\mathbb{N}}{\pi}}$ & 33,1 & 37,1 & 39,1 & 40,4 & 33,8 & 26,6 & 19,9 \\
\hline
\end{tabular}

Tabla 13. Tasa bruta población que se gradúa $(2013 / 14)$.

\begin{tabular}{|c|c|c|c|}
\hline 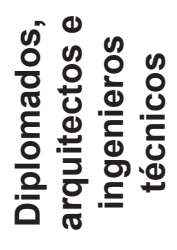 & $\begin{array}{l}\stackrel{0}{0} \\
\frac{0}{0} \\
\frac{\pi}{0} \\
\frac{0}{0} \\
\frac{0}{J}\end{array}$ & $\begin{array}{l}\frac{0}{0} \\
\frac{\pi}{0}\end{array}$ & 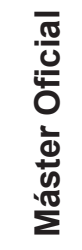 \\
\hline 4,5 & 15,3 & 30,1 & 14,0 \\
\hline 4,3 & 14,4 & 17,6 & 9,3 \\
\hline 6,0 & 17,1 & 26,6 & 7,3 \\
\hline 8,1 & 15,5 & 21,2 & 9,1 \\
\hline 1,8 & 2,4 & 13,2 & 5,5 \\
\hline 3,5 & 7,6 & 14,1 & 3,1 \\
\hline 4,8 & 13,4 & 18,6 & 7,6 \\
\hline 6,1 & 21,7 & 63,0 & 18,7 \\
\hline 0,8 & 4,0 & 18,7 & 2,7 \\
\hline 1,8 & 10,1 & 31,0 & 12,5 \\
\hline
\end{tabular}




\begin{tabular}{|c|c|c|c|c|c|c|c|c|c|c|c|}
\hline 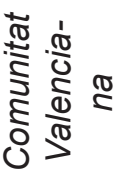 & 30,2 & 34,1 & 37,2 & 38,4 & 34,8 & 27,3 & 21,5 & 5,1 & 18,5 & 27,7 & 15,6 \\
\hline 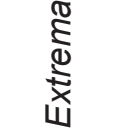 & 17,3 & 20,4 & 23,0 & 25,0 & 20,8 & 16,6 & 12,9 & 5,4 & 5,2 & 15,9 & 5,6 \\
\hline$\frac{.0}{0}$ & 27,4 & 30,3 & 32,8 & 32,7 & 29,7 & 25,3 & 19,4 & 5,0 & 13,6 & 22,7 & 13,2 \\
\hline 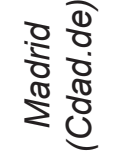 & 49,6 & 55,3 & 56,5 & 56,4 & 49,4 & 40,5 & 29,6 & 4,9 & 23,8 & 49,2 & 20,0 \\
\hline 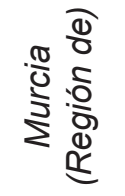 & 30,1 & 34,6 & 37,1 & 37,3 & 30,7 & 25,5 & 19,2 & 2,4 & 8,1 & 36,6 & 13,3 \\
\hline $\begin{array}{l}\frac{\pi}{0} \\
\frac{\pi}{0} \frac{0}{0} \\
\frac{\pi}{0}\end{array}$ & 35,6 & 38,7 & 40,5 & 40,6 & 31,2 & 24,7 & 15,3 & 3,5 & 14,4 & 34,5 & 20,7 \\
\hline $\begin{array}{l}0 \\
0 \\
\frac{1}{0} \\
\frac{0}{\pi} \\
0\end{array}$ & 44,2 & 45,5 & 48,1 & 47,7 & 34,8 & 27,3 & 17,8 & 5,4 & 16,2 & 35,5 & 14,3 \\
\hline 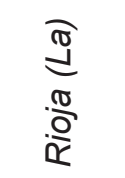 & 20,0 & 22,1 & 26,2 & 26,4 & 35,0 & 33,4 & 33,2 & 5,4 & 12,2 & 16,5 & 3,3 \\
\hline
\end{tabular}

Fuente: Ministerio de Educación, Cultura y Deporte. - No se incluyen Ceuta y Melilla por su escasa representatividad. 
Tabla 14. Alumnado que terminó los estudios.

\begin{tabular}{|c|c|c|c|c|}
\hline & 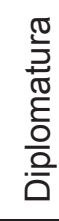 & $\begin{array}{l}\frac{\sigma}{J} \\
\stackrel{0}{0} \\
\frac{\pi}{0} \\
\frac{0}{0} \\
. \frac{0}{J}\end{array}$ & $\begin{array}{l}\frac{0}{0} \\
\frac{\pi}{0} \\
0\end{array}$ & 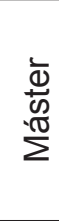 \\
\hline $\begin{array}{l}\frac{1}{\leqslant} \\
\stackrel{0}{\circ}\end{array}$ & $\stackrel{\infty}{\check{E}}$ & $\begin{array}{l}\mathscr{D} \\
\stackrel{0}{0} \\
\stackrel{N}{N}\end{array}$ & 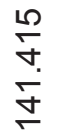 & 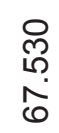 \\
\hline$\frac{\frac{\pi}{0}}{\frac{0}{\frac{\pi}{\pi}}}$ & $\begin{array}{l}\text { \& } \\
\text { O } \\
\dot{+}\end{array}$ & $\begin{array}{l}\stackrel{N}{\sim} \\
\stackrel{+}{ \pm}\end{array}$ & $\begin{array}{l}\stackrel{\infty}{N} \\
\stackrel{N}{\leftarrow}\end{array}$ & $\begin{array}{l}\bar{P} \\
\text { ஸे }\end{array}$ \\
\hline 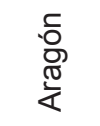 & $\underset{N}{\mathbb{N}}$ & 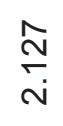 & $\begin{array}{l}\stackrel{0}{\circ} \\
\text { mे } \\
\text { m. }\end{array}$ & స్ \\
\hline 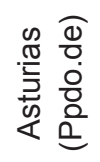 & 웅 & 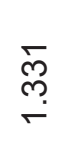 & 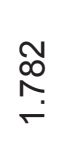 & $\stackrel{\infty}{\infty}$ \\
\hline 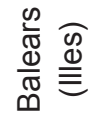 & Бু & $\begin{array}{l}\infty \\
\stackrel{\infty}{\sim}\end{array}$ & $\stackrel{\Gamma}{\stackrel{5}{r}}$ & $\stackrel{\infty}{\stackrel{0}{0}}$ \\
\hline $\begin{array}{l}\frac{0}{0} \\
\frac{\pi}{0} \\
\frac{\pi}{\pi} \\
\frac{\pi}{\pi} \\
0\end{array}$ & ְొ & $\begin{array}{l}\stackrel{L}{0} \\
\stackrel{0}{\stackrel{2}{r}}\end{array}$ & $\begin{array}{l}0 \\
\stackrel{0}{0} \\
\text { m }\end{array}$ & $\stackrel{\bullet}{\stackrel{D}{R}}$ \\
\hline 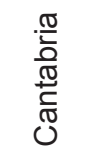 & $\stackrel{్}{N}$ & $\overline{8}$ & ஜ্ & $\frac{0}{\dot{y}}$ \\
\hline 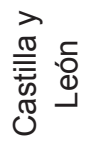 & 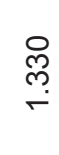 & 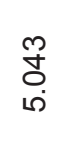 & $\begin{array}{l}\stackrel{0}{m} \\
\stackrel{m}{\leftarrow}\end{array}$ & \begin{tabular}{l}
\multirow{o}{+}{} \\
$\stackrel{+}{+}$
\end{tabular} \\
\hline 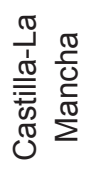 & $\underset{\infty}{\infty}$ & ర్లి & $\begin{array}{l}\text { ô } \\
\stackrel{m}{\sim} \\
\sim\end{array}$ & ஜ్ \\
\hline 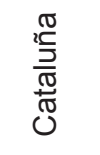 & $\stackrel{\stackrel{\leftrightarrow}{\leftrightarrow}}{\stackrel{\leftrightarrow}{\leftarrow}}$ & $\begin{array}{l}\infty \\
\stackrel{8}{\circ} \\
\stackrel{2}{*}\end{array}$ & 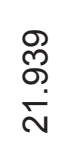 & 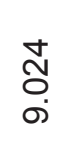 \\
\hline 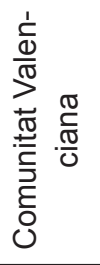 & 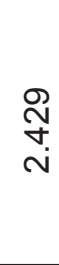 & 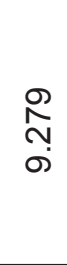 & $\begin{array}{l}\stackrel{m}{\infty} \\
\stackrel{\infty}{m}\end{array}$ & $\stackrel{\infty}{\stackrel{+}{\sigma}}$ \\
\hline
\end{tabular}

Tabla 15. \% población de 30-34 años con estudios superiores.

\begin{tabular}{|c|c|c|c|}
\hline & ஜ̊ & $\stackrel{\circ}{\stackrel{2}{\alpha}}$ & $\stackrel{\infty}{\stackrel{N}{N}}$ \\
\hline 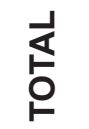 & ब্ & $\begin{array}{l}\stackrel{0}{\text { v }} \\
\text { Tे }\end{array}$ & $\begin{array}{l}\text { ̊) } \\
\text { ơ }\end{array}$ \\
\hline $\begin{array}{l}\frac{\pi}{0} \\
\frac{3}{3} \\
\frac{\pi}{2} \\
\frac{1}{4}\end{array}$ & $\frac{\vec{m}}{\dot{m}}$ & $\begin{array}{c}m \\
w^{-}\end{array}$ & జ్లు \\
\hline 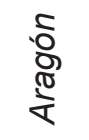 & $\overline{\widetilde{m}}$ & $\begin{array}{l}m \\
\theta^{-}\end{array}$ & 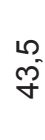 \\
\hline 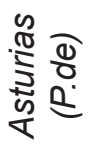 & $\begin{array}{c}N \\
\text { ó } \\
\dot{q}\end{array}$ & $\begin{array}{l}\stackrel{\leftrightarrow}{\sim} \\
\stackrel{\sim}{*}\end{array}$ & $\begin{array}{l}\text { o } \\
\text { Ni }\end{array}$ \\
\hline 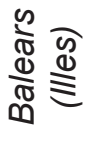 & $\hat{0}$ & $\begin{array}{l}\stackrel{0}{0} \\
\mathbb{N}^{\circ}\end{array}$ & $\overline{\mathrm{N}}$ \\
\hline 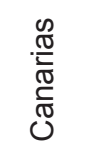 & $\stackrel{0}{\tilde{m}^{-}}$ & $\bar{m}$ & $\begin{array}{l}\text { న } \\
\text { ని }\end{array}$ \\
\hline 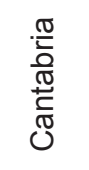 & $\begin{array}{l}\text { ஜे } \\
\text { ซ্ }\end{array}$ & $\begin{array}{l}m \\
\text { f }\end{array}$ & $\frac{10}{\sigma}$ \\
\hline 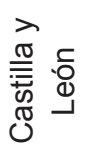 & $\frac{5}{\sigma}$ & $\begin{array}{l}0 \\
\dot{q} \\
\dot{q}\end{array}$ & $\overline{\mathcal{J}}$ \\
\hline 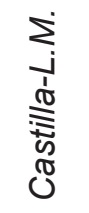 & $\overline{\bar{m}^{\prime}}$ & $\hat{\bar{m}}$ & $\stackrel{N}{\tilde{m}}$ \\
\hline 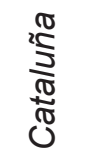 & $\stackrel{N}{\leftarrow}$ & $\hat{\widetilde{Y}}$ & $\bar{m}$ \\
\hline 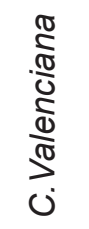 & 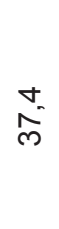 & $\begin{array}{l}0 \\
\infty^{\circ}\end{array}$ & $\hat{o}$ \\
\hline
\end{tabular}




\begin{tabular}{|c|c|c|c|c|c|c|c|c|}
\hline 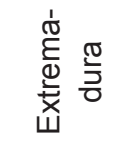 & $\underset{0}{\mathbb{6}}$ & $\begin{array}{l}\text { N } \\
0 \\
0\end{array}$ & 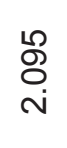 & 莡 & 离 & $\stackrel{0}{\tilde{m}}$ & 足 & $\begin{array}{l}0 \\
\dot{q} \\
\stackrel{q}{0}\end{array}$ \\
\hline $\begin{array}{l}\frac{\pi}{0} \\
\frac{.0}{\pi} \\
0\end{array}$ & $\stackrel{8}{\frac{8}{7}}$ & $\begin{array}{l}\stackrel{Ð}{N} \\
\text { mे } \\
m\end{array}$ & \begin{tabular}{l}
$\hat{\infty}$ \\
\multirow{2}{*}{} \\
in
\end{tabular} & $\underset{m}{\stackrel{\nabla}{m}}$ & $\frac{\pi}{0}$ & $\begin{array}{l}0 \\
\text { ó }\end{array}$ & $\stackrel{+}{\underset{f}{*}}$ & $\begin{array}{l}\infty \\
\stackrel{\infty}{f}\end{array}$ \\
\hline 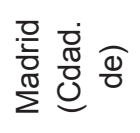 & $\begin{array}{l}\mathscr{D} \\
\infty \\
\infty \\
\sim\end{array}$ & $\begin{array}{l}\underset{⿱}{\mathbb{Z}} \\
\stackrel{+}{\leftarrow}\end{array}$ & $\begin{array}{l}\text { مी } \\
\text { مी } \\
0\end{array}$ & 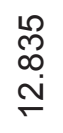 & 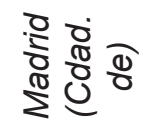 & $\frac{0}{5}$ & $\stackrel{\circ}{\text { లై }}$ & $\begin{array}{l}\infty \\
\text { ¿ } \\
\text { in }\end{array}$ \\
\hline 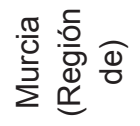 & প్ల & ஸ̂ & $\stackrel{\infty}{\stackrel{0}{0}}$ & $\begin{array}{l}\infty \\
\vdots \\
\stackrel{N}{N}\end{array}$ & 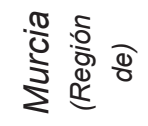 & $\frac{\Delta}{m}$ & $\begin{array}{l}\text { M. } \\
\text { Dे }\end{array}$ & 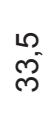 \\
\hline 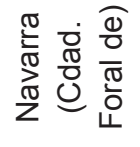 & : & ঃి & 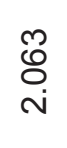 & 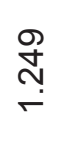 & 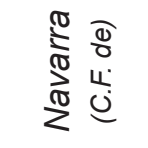 & $\begin{array}{c}m \\
\stackrel{\infty}{\infty^{\circ}}\end{array}$ & $\begin{array}{l}0 \\
\text { in }\end{array}$ & $\stackrel{\stackrel{9}{*}}{\stackrel{f}{*}}$ \\
\hline 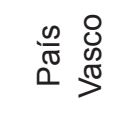 & ల్ల & $\begin{array}{l}\bigotimes \\
\infty \\
i \\
i\end{array}$ & 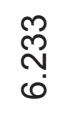 & 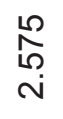 & 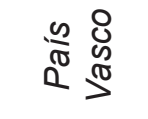 & $\begin{array}{l}\infty \\
\stackrel{\circ}{\circ} \\
\stackrel{n}{n}\end{array}$ & $\begin{array}{l}\infty \\
\text { on }\end{array}$ & $\begin{array}{l}N \\
\text { Dే }\end{array}$ \\
\hline 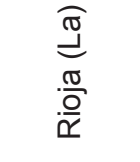 & $\stackrel{50}{5}$ & ల్లి & $\stackrel{\mathscr{P}}{\stackrel{\sim}{\sim}}$ & 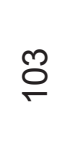 & $\frac{\pi}{2} \frac{\pi}{d}$ & $\overline{\tilde{N}}$ & $\begin{array}{l}\hat{\infty} \\
\operatorname{or}^{-}\end{array}$ & F- \\
\hline 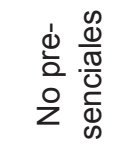 & 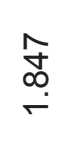 & $\frac{\hat{\sigma}}{\dot{\sigma}}$ & $\begin{array}{l}\infty \\
\infty \\
\infty \\
i\end{array}$ & $\begin{array}{l}\hat{1} \\
6 \\
0\end{array}$ & & & & \\
\hline
\end{tabular}


Tabla 17. \% Distribución del profesorado de las universidades públicas (2014-15).

\begin{tabular}{|c|c|c|c|}
\hline & 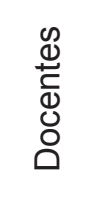 & 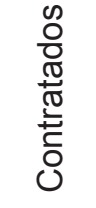 & 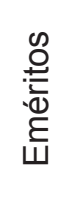 \\
\hline TOTAL & 46,1 & 53,3 & 0,6 \\
\hline A Coruña & 51,0 & 48,9 & 0,1 \\
\hline Alcalá de Henares & 44,2 & 52,5 & 3,3 \\
\hline Alicante & 42,5 & 57,2 & 0,3 \\
\hline Almería & 65,1 & 34,7 & 0,1 \\
\hline Aut.de Barcelona & 28,8 & 70,0 & 1,2 \\
\hline Autónoma de Madrid & 46,9 & 53,1 & 0,0 \\
\hline Barcelona & 37,5 & 61,7 & 0,8 \\
\hline Burgos & 41,8 & 57,4 & 0,8 \\
\hline Cádiz & 46,6 & 53,4 & 0,0 \\
\hline Cantabria & 40,8 & 58,8 & 0,4 \\
\hline Carlos III & 38,9 & 60,8 & 0,3 \\
\hline Castilla-La Mancha & 41,7 & 58,3 & 0,0 \\
\hline Complutense Madrid & 46,2 & 53,4 & 0,5 \\
\hline Córdoba & 47,0 & 53,0 & 0,0 \\
\hline Extremadura & 45,0 & 54,8 & 0,2 \\
\hline Girona & 28,8 & 71,0 & 0,1 \\
\hline Granada & 59,2 & 40,5 & 0,3 \\
\hline Huelva & 52,4 & 47,6 & 0,0 \\
\hline Illes Balears & 33,8 & 64,9 & 1,3 \\
\hline Jaén & 61,1 & 38,9 & 0,0 \\
\hline Jaume I de Castellón & 39,3 & 60,7 & 0,1 \\
\hline La Laguna & 61,7 & 38,3 & 0,0 \\
\hline La Rioja & 56,1 & 43,9 & 0,0 \\
\hline Las Palmas de G.C. & 51,6 & 47,7 & 0,7 \\
\hline León & 60,0 & 39,8 & 0,2 \\
\hline Lleida & 32,0 & 66,2 & 1,7 \\
\hline Málaga & 50,4 & 49,3 & 0,4 \\
\hline $\begin{array}{l}\text { Miguel Hdez. de } \\
\text { Elche }\end{array}$ & 31,4 & 68,3 & 0,4 \\
\hline Murcia & 44,6 & 54,8 & 0,6 \\
\hline Oviedo & 66,2 & 33,7 & 0,1 \\
\hline Pablo de Olavide & 26,5 & 73,5 & 0,0 \\
\hline
\end{tabular}

Tabla 16. Tasa bruta de población que supera la prueba de acceso a la universidad.

\begin{tabular}{|l|l|l|l|}
\hline & & & \\
& 2005 & 2010 & 2015 \\
\hline \multicolumn{1}{|c|}{ TOTAL } & 37,2 & 40,4 & 51,8 \\
Andalucía & 30,4 & 35,8 & 50,7 \\
Aragón & 42,3 & 43,3 & 54,8 \\
Asturias (Ppado. de) & 46,3 & 51,9 & 48,3 \\
Balears (IIles) & 29,4 & 32,7 & 38,8 \\
Canarias & 33,1 & 38,5 & 50,2 \\
Cantabria & 36,2 & 41,3 & 53,6 \\
Castilla y León & 40,4 & 45,2 & 55,4 \\
Castilla-La Mancha & 35,1 & 36,1 & 46,3 \\
Cataluña & 34,4 & 40,2 & 47,0 \\
Comunitat Valenciana & 36,0 & 37,0 & 48,3 \\
Extremadura & 36,6 & 40,4 & 54,0 \\
Galicia & 38,9 & 38,3 & 54,5 \\
Madrid (Cdad.de) & 40,5 & 43,3 & 54,1 \\
Murcia (Región de) & 32,0 & 39,9 & 49,0 \\
Navarra (Cdad. Foral de) & 39,6 & 44,1 & 51,1 \\
País Vasco & 50,2 & 53,3 & 66,1 \\
Rioja (La) & 39,6 & 40,7 & 48,4 \\
\hline
\end{tabular}

Fuente: Ministerio de Educación, Cultura y Deporte.

No se incluyen Ceuta y Melilla por escasa representatividad. No se tiene en cuenta las Pruebas para >25, 45 y 40 años. 


\begin{tabular}{|l|ccc|}
\hline País Vasco & 45,3 & 54,5 & 0,2 \\
$\begin{array}{l}\text { Politécnica de } \\
\text { Cartagena }\end{array}$ & 45,8 & 54,2 & 0,0 \\
$\begin{array}{l}\text { Politécnica de } \\
\text { Cataluña }\end{array}$ & 43,5 & 56,5 & 0,0 \\
Politécnica de & & & \\
Madrid & 65,7 & 33,6 & 0,7 \\
Politécnica de Valencia & 59,1 & 40,7 & 0,2 \\
Pompeu Fabra & 18,8 & 78,7 & 2,4 \\
Pública de Navarra & 39,6 & 60,3 & 0,1 \\
Rey Juan Carlos & 39,7 & 60,3 & 0,0 \\
Rovira i Virgili & 25,9 & 74,1 & 0,0 \\
Salamanca & 47,8 & 52,2 & 0,0 \\
Santiago & 61,9 & 38,1 & 0,0 \\
Sevilla & 47,9 & 51,7 & 0,3 \\
UNED & 51,7 & 45,9 & 2,4 \\
Valencia & 48,2 & 50,9 & 0,9 \\
(Est. General) & 53,0 & 46,9 & 0,1 \\
Valladolid & 50,3 & 49,5 & 0,2 \\
Vigo & 41,7 & 53,6 & 4,7 \\
\hline Zaragoza & & \\
\hline
\end{tabular}

Fuente: Ministerio de Educación, Cultura y Deporte 
En relación a los modelos de relaciones laborales del profesorado, para concretar la diversidad de tipos de contratación que permita detectar los niveles de estabilidad, se han englobado en tres grupos: docentes, contratados y eméritos. En el curso 2014/15 (Tabla-17), la mayoría del profesorado universitario español era contratado $(53,3 \%)$ y un $46,1 \%$ eran docentes propiamente dichos. Sin embargo, ambas universidades públicas canarias muestran un alto índice de estabilidad en sus plantillas, pues en La Laguna el $62 \%$ de sus profesores eran docentes y en Las Palmas de Gran Canaria el $52 \%{ }^{2}$.

- IV.- Los resultados. Sería interminable detallar la gran cantidad de datos que arroja la educación universitaria en España; pero un exceso de concreción podría desvirtuar el debido análisis objetivo e introducir falacias lógicas, especialmente las indicadas por Sodaro (2010: 46-47) como falacias de composición al considerar que el todo sea igual que las partes que lo componen, o falacias de falsa inferencia por alcanzar indebidamente una serie de conclusiones en base a datos estadísticos aislados. Por ello, en la selección se han tenido en cuenta estos aspectos para poder plantear las conclusiones eficazmente, cuyos mejores exponentes son las Tablas 18 y 19 que se explicarán más adelante

Los beneficios que supone contar con titulación superior para mejorar los ingresos, corren paralelos a un incremento de la sensibilidad entre los titulados universitarios hacia la colaboración en proyectos sociales, así como por su participación política y ciudadana:

1. El $47,5 \%$ de la población con ingresos por hogar superiores a los 35.000 euros, tiene formación superior. Pero con ese nivel retributivo sólo se encuentran el $23 \%$ de los que han finalizado la segunda etapa de Educación Secundaria, el 17,7\% de los que alcanzaron la primera etapa de Educación Secundaria, y el 11,8\% de los que apenas disponen de Educación Primaria o inferior (Tabla-21). El porcentaje de participación de los titulados superiores en los grupos de población según sus ingresos, desciende en línea con cada menor rango retributivo.

2. Las personas que disponen de educación superior son las que más participan en acciones de voluntariado, tanto al margen de organizaciones dedicadas a estos fines como dentro de ellas (Tabla-20).

3. Un $13,8 \%$ de los titulados superiores se deciden a participar en partidos políticos o manifestaciones públicas de protesta, frente a un $9,5 \%$ de los que han finalizado la segunda etapa de Educación Secundaria en España, un 5,2\% de los que alcanzaron la primera etapa de secundaria, y un 2,6\% de aquellos con Educación Primaria o inferior (Tabla-22).

Los datos internacionales sitúan a España en una situación de privilegio en cuanto a los resultados académicos, pese a la menor inversión pública relativa. Los españoles de 30 a 34 años con formación superior han crecido un punto entre 2005 y 2015 hasta situarse en el 40,9\%, por encima de la media de la UE que está en el 38,8\% (Tabla-24). Cabría la tentación de matizar este dato atendiendo al efecto estadístico tras la incorporación de los países del Este europeo, especialmente teniendo en cuenta que en Estados como Francia o el Reino Unido la población de esta franja de edad con formación universitaria es del $45 \%$ y 47,9\%, respectivamente; pero en Alemania es del 32,3\%, en Austria del $38,7 \%$ y en Italia del $25,3 \%$ - todos ellos por debajo de España -, y naciones como Letonia o Lituania están por encima del dato español (con un $41,3 \%$ y un $57,6 \%$, respectivamente). Por lo que España muestra un aceptable resultado en esta variable, pese a que su gasto público en educación superior representa el 0,97\% del PIB (Tabla-23), muy por debajo (en este caso sí) de la media comunitaria, que se eleva hasta el 1,29\% (con porcentajes superiores al $2 \%$ en países como Suecia o Finlandia). En ámbitos como la formación permanente de adultos los resultados no son tan halagüeños, pues sólo un 9,9\% de los españoles entre 25 y 64 años participaba en 2015 en la mejora constante de sus conocimientos (Tabla-25), lo que supone una caída de 1,3 puntos en comparación al año 2010 y deja a España por debajo de la media comunitaria. Es preciso indicar que en España casi el 76\% de los titulados universitarios estaban trabajando en el año 2014 (Tabla-26); pero en Canarias menos del $67 \%$ de los que poseían educación superior ocupaban un puesto de trabajo, lo que sitúa a dicha comunidad en último puesto nacional. 
Tabla 20. \% Personas > 16 años que participan en voluntariado por nivel de formación (2015).

\begin{tabular}{|c|c|c|c|c|c|c|c|c|c|c|}
\hline & \multicolumn{5}{|c|}{ Al margen de organizaciones de estos fines } & \multicolumn{5}{|c|}{ A través de una organización, asociación o agrupación } \\
\hline & $\begin{array}{l}\overline{\widetilde{\pi}} \\
\stackrel{0}{0}\end{array}$ & 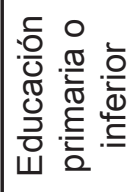 & 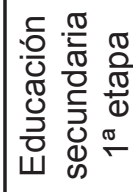 & 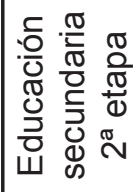 & 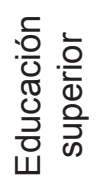 & $\begin{array}{l}\bar{\pi} \\
\stackrel{0}{0}\end{array}$ & 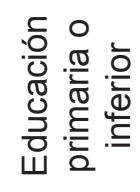 & 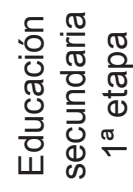 & 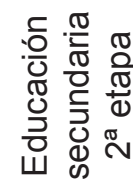 & 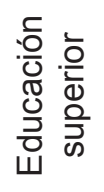 \\
\hline $\bar{\omega}$ & 10,6 & 6,1 & 9,7 & 11,3 & 15,1 & 10,7 & 5,9 & 8,4 & 11,7 & 16,5 \\
\hline 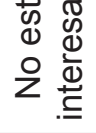 & 14,3 & 17,6 & 18,0 & 13,4 & 8,7 & 14,1 & 17,2 & 18,0 & 13,1 & 8,5 \\
\hline 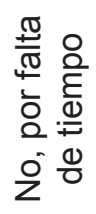 & 18,9 & 6,9 & 17,3 & 24,0 & 27,4 & 20,7 & 8,5 & 19,1 & 26,0 & 29,3 \\
\hline 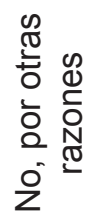 & 56,1 & 69,4 & 55,0 & 51,2 & 48,9 & 54,4 & 68,5 & 54,5 & 49,2 & 45,6 \\
\hline
\end{tabular}

Tabla 21. \% Distribución según nivel de formación por ingresos del hogar (2015).

\begin{tabular}{|c|c|c|c|c|c|}
\hline & 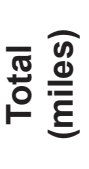 & 竞 & 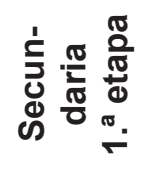 & 它 & 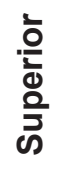 \\
\hline $\begin{array}{l}\bar{\pi} \\
\text { ठే }\end{array}$ & $\begin{array}{l}\hat{\Sigma} \\
\text { No } \\
\infty \\
\infty \\
\infty\end{array}$ & ڤึ & $\begin{array}{l}\stackrel{0}{\sim} \\
\stackrel{\text { N }}{n}\end{array}$ & $\frac{N}{\grave{N}}$ & م \\
\hline 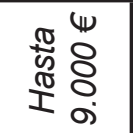 & $\begin{array}{l}\text { D) } \\
\infty \\
\dot{m}\end{array}$ & $\begin{array}{l}\text { م) } \\
\text { लै }\end{array}$ & $\begin{array}{l}\dot{\nabla} \\
\dot{m}\end{array}$ & $\begin{array}{l}\circ \\
\stackrel{2}{\sim}\end{array}$ & $\frac{5}{2}$ \\
\hline 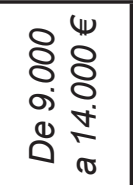 & $\begin{array}{l}\infty \\
\stackrel{\infty}{\sigma} \\
\forall\end{array}$ & 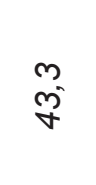 & $\begin{array}{l}\stackrel{N}{ } \\
\stackrel{\sim}{\sim}\end{array}$ & $\stackrel{m}{0}$ & 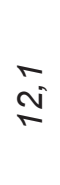 \\
\hline 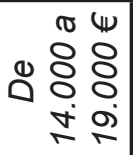 & $\begin{array}{l}\frac{1}{d} \\
\text { in }\end{array}$ & $\begin{array}{l}\text { م) } \\
\text { ले }\end{array}$ & $\begin{array}{l}\infty \\
\text { Dे }\end{array}$ & $\begin{array}{l}m \\
\stackrel{9}{r}\end{array}$ & $\begin{array}{l}0 \\
0^{\circ} \\
\end{array}$ \\
\hline 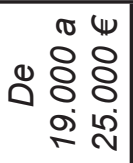 & \begin{tabular}{l}
$\forall$ \\
\multirow{0}{0}{} \\
0
\end{tabular} & $\begin{array}{l}\infty \\
\text { }^{-}\end{array}$ & $\begin{array}{l}0 \\
\stackrel{D}{N}\end{array}$ & $\begin{array}{l}m \\
\stackrel{N}{N}\end{array}$ & 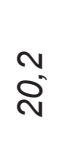 \\
\hline 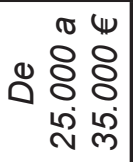 & 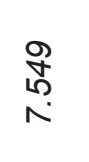 & $\begin{array}{l}\forall \\
\stackrel{N}{*}\end{array}$ & 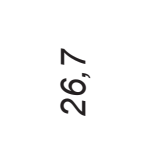 & $\begin{array}{l}\text { Dे } \\
\text { D }\end{array}$ & $\begin{array}{l}\text { o } \\
\infty^{\circ}\end{array}$ \\
\hline 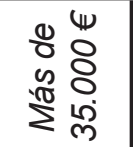 & $\begin{array}{l}\stackrel{\infty}{N} \\
\stackrel{N}{=}\end{array}$ & $\begin{array}{l}\infty \\
=\end{array}$ & $\hat{N}$ & $\begin{array}{l}\circ \\
\text { N }\end{array}$ & 尔 \\
\hline
\end{tabular}

Tabla 22. \% Personas mayores de 16 años que participan en partidos políticos, manifestaciones, etc., por nivel de formación (2015).

\begin{tabular}{|c|c|c|}
\hline & Total & $\begin{array}{c}\text { Primaria o } \\
\text { inferior }\end{array}$ \\
\hline Sí & 7,9 & 2,6 \\
\hline $\begin{array}{c}\text { No está } \\
\text { interesado }\end{array}$ & 52,5 & 50,4 \\
\hline $\begin{array}{c}\text { No, por } \\
\text { falta de tiempo }\end{array}$ & 4,7 & 1,9 \\
\hline $\begin{array}{l}\text { No, por otras } \\
\text { razones }\end{array}$ & 34,9 & 45,1 \\
\hline
\end{tabular}

Fuente: Ministerio de Educación, Cultura y Deporte. 
Tabla 23. \% PIB gasto público en educación superior (2013).
Tabla 24. \% Población 30 a 34 años que ha alcanzado formación superior.
Tabla 25. \% Población 25 a 64 años que participa en formación permanente.

\begin{tabular}{|c|c|c|c|c|c|c|c|c|c|}
\hline UE-28 & 1,29 & & 2005 & 2010 & 2015 & & 2005 & 2010 & 2015 \\
\hline Alemania & 1,35 & UE-28 & 28,1 & 33,8 & 38,7 & UE-28 & 9,6 & 9,3 & 10,7 \\
\hline Austria & 1,86 & Alemania & 26,1 & 29,7 & 32,3 & Alemania & 7,7 & 7,8 & 8,1 \\
\hline Bélgica & 1,49 & Austria & 20,7 & 23,4 & 38,7 & Austria & 12,9 & 13,8 & 14,4 \\
\hline Bulgaria & 0,68 & Bélgica & 39,1 & 44,4 & 42,7 & Bélgica & 8,3 & 7,4 & 6,9 \\
\hline Chipre & 1,58 & Bulgaria & 24,9 & 28,0 & 32,1 & Bulgaria & 1,3 & 1,6 & 2,0 \\
\hline Croacia & .. & Chipre & 40,8 & 45,3 & 54,5 & Chipre & 5,9 & 8,1 & 7,5 \\
\hline Dinamarca & .. & Croacia & 17,4 & 24,5 & 30,9 & Croacia & 2,1 & 3,0 & 3,1 \\
\hline Eslovenia & 1,15 & Dinamarca & 43,1 & 41,2 & 47,6 & Dinamarca & 27,4 & 32,6 & 31,3 \\
\hline España & 0,97 & Eslovenia & 24,6 & 34,8 & 43,4 & Eslovenia & 15,3 & 16,4 & 11,9 \\
\hline Estonia & 1,39 & España & 39,9 & 42,0 & 40,9 & España & 10,8 & 11,2 & 9,9 \\
\hline Finlandia & 2,11 & Estonia & 31,7 & 40,2 & 45,3 & Estonia & 6,0 & 11,0 & 12,4 \\
\hline Francia &.. & Finlandia & 43,7 & 45,7 & 45,5 & Finlandia & 22,5 & 23,0 & 25,4 \\
\hline Grecia & .. & Francia & 37,7 & 43,2 & 45,0 & Francia & 5,9 & 5,0 & 18,6 \\
\hline Hungría & 0,93 & Grecia & 25,5 & 28,6 & 40,4 & Grecia & 1,9 & 3,3 & 3,3 \\
\hline Irlanda & 1,26 & Hungría & 17,9 & 26,1 & 34,3 & Hungría & 3,9 & 3,0 & 7,1 \\
\hline Italia & 0,84 & Irlanda & 39,2 & 50,1 & 52,3 & Irlanda & 7,4 & 7,0 & 6,5 \\
\hline Letonia & 0,66 & Italia & 17,1 & 19,9 & 25,3 & Italia & 5,8 & 6,2 & 7,3 \\
\hline Lituania & 1,34 & Letonia & 18,5 & 32,6 & 41,3 & Letonia & 7,8 & 5,4 & 5,7 \\
\hline Luxemburgo & .. & Lituania & 37,7 & 43,8 & 57,6 & Lituania & 6,1 & 4,4 & 5,8 \\
\hline Malta & 1,60 & Luxemburgo & 37,6 & 46,1 & 52,3 & Luxemburgo & 8,5 & 13,5 & 18,0 \\
\hline Países Bajos & 1,75 & Malta & 17,6 & 22,1 & 27,8 & Malta & 5,2 & 6,2 & 7,2 \\
\hline Polonia & 1,22 & Países Bajos & 34,9 & 41,4 & 46,3 & Países Bajos & 15,9 & 17,0 & 18,9 \\
\hline Portugal & 0,92 & Polonia & 22,7 & 34,8 & 43,4 & Polonia & 4,9 & 5,2 & 3,5 \\
\hline Reino Unido & 1,56 & Portugal & 17,5 & 24,0 & 31,9 & Portugal & 4,1 & 5,7 & 9,7 \\
\hline República Checa & 0,93 & Reino Unido & 34,5 & 43,1 & 47,9 & Reino Unido & 27,6 & 20,1 & 15,7 \\
\hline $\begin{array}{l}\text { República } \\
\text { Eslovaca }\end{array}$ & 0,99 & R. Checa & 13,0 & 20,4 & 30,1 & R. Checa & 5,6 & 7,8 & 8,5 \\
\hline Rumania & 0,73 & R. Eslovaca & 14,3 & 22,1 & 28,4 & R. Eslovaca & 4,6 & 3,1 & 3,1 \\
\hline Suecia & 2,03 & Rumanía & 11,4 & 18,3 & 25,6 & Rumanía & 1,6 & 1,4 & 1,3 \\
\hline Islandia & 1,59 & Suecia & 37,6 & 45,3 & 50,2 & Suecia & 17,4 & 24,7 & 29,4 \\
\hline Noruega & 1,95 & Islandia & 41,1 & 40,9 & 47,1 & Islandia & 25,7 & 25,4 & 28,1 \\
\hline Suiza & 1,40 & Noruega & 39,4 & 47,3 & 50,9 & Noruega & 17,8 & 18,2 & 20,1 \\
\hline Turquía & 1,59 & Suiza & 33,4 & 44,2 & 51,4 & Suiza & 27,0 & 30,6 & 32,1 \\
\hline
\end{tabular}

Fuente: M. ${ }^{\circ}$ Educación Fuente: M. ${ }^{\circ}$ Educación (Encuesta Europea Población Activa 2016).

(Cuestionario UOE) 
Tabla 26. Titulados universitarios según su situación laboral en 2014.

\begin{tabular}{|l|c|c|c|}
\hline & Trabajando & En desempleo & Inactivo \\
\hline Total nacional & 75,6 & 18,0 & 6,4 \\
Andalucía & 67,5 & 26,1 & 6,5 \\
Aragón & 80,9 & 12,8 & 6,3 \\
Asturias, Principado de & 70,8 & 21,9 & 7,4 \\
Balears, Illes & 81,1 & 12,6 & 6,4 \\
Canarias & $\mathbf{6 6 , 9}$ & $\mathbf{2 3 , 6}$ & $\mathbf{9 , 5}$ \\
Cantabria & 73,8 & 21,2 & 5,0 \\
Castilla y León & 73,4 & 20,2 & 6,3 \\
Castilla - La Mancha & 70,2 & 24,0 & 5,8 \\
Cataluña & 82,8 & 11,0 & 6,1 \\
Comunitat Valenciana & 73,3 & 20,2 & 6,5 \\
Extremadura & 69,1 & 25,2 & 5,7 \\
Galicia & 72,5 & 21,4 & 6,1 \\
Madrid, Comunidad de & 80,7 & 13,6 & 5,6 \\
Murcia, Región de & 72,3 & 20,0 & 7,7 \\
Navarra, Cdad.Foral de & 78,4 & 14,4 & 7,2 \\
País Vasco & 78,5 & 16,0 & 5,5 \\
Rioja, La & 80,4 & 11,5 & 8,1 \\
Universidades no presenciales & 76,3 & 13,2 & 10,5 \\
\hline
\end{tabular}

Fuente: Instituto Nacional de Estadística.

Se indica la Comunidad Autónoma de la Universidad donde se estudió.

\subsection{Evolución del empleo universitario}

El informe sobre la Modernización de la Educación Superior en Europa de 2017, concluye que «La seguridad en el empleo ha dejado de ser la norma en el mundo académico» (Comisión Europea, 2017: 9). Pero las ventajas en los salarios de las personas con estudios superiores en España está demostrada por la multitud de estudios realizados, que pueden resumirse en que "ganan un $40 \%$ más que las que han finalizado la segunda etapa de Educación Secundaria y un $60 \%$ más que las que han completado la primera etapa de Educación Secundaria o un nivel inferior» (Ministerio de Educación, Cultura y Deporte, 2016b: 35). La Tabla-18 muestra que durante la crisis la preocupación por mejorar la formación es creciente:

Tabla 18. Ocupados que cursan estudios (reglados o no) por nivel de formación.

\begin{tabular}{|l|c|c|c|c|c|}
\hline \multicolumn{1}{|c|}{ (miles de personas) } & $\mathbf{2 0 1 7 T 3}$ & $\mathbf{2 0 1 6 T 1}$ & $2015 \mathrm{~T} 1$ & $2014 \mathrm{~T} 1$ & 2017 vs 2014 \\
\hline \multicolumn{1}{|c}{ Total } & $1.572,0$ & $1.960,1$ & $1.979,7$ & $2.023,5$ & $-22,3 \%$ \\
Analfabetos & 0,2 & 2,7 & 1,3 & 0,9 & $-77,8 \%$ \\
Estudios primarios incompletos & 2,0 & 2,9 & 1,5 & 2,7 & $-25,9 \%$ \\
Educación Primaria & 16,9 & 16,2 & 22,1 & 37,2 & $-54,6 \%$ \\
$1^{\text {a }}$ etapa Educación Secundaria y similar & 184,7 & 210,0 & 219,9 & 232,4 & $-20,5 \%$ \\
$2^{\mathrm{a}}$ etapa Secundaria (orientación general) & 318,4 & 290,5 & 285,1 & 290,6 & $9,6 \%$ \\
$2^{\mathrm{a}}$ etapa Secundaria (orientación profesional) & 126,5 & 140,3 & 139,1 & 126,9 & $-0,3 \%$ \\
Educación superior & 923,2 & $1.297,6$ & $1.310,8$ & $1.332,8$ & $-30,7 \%$ \\
\hline
\end{tabular}

Fuente: Instituto Nacional de Estadística - Elaboración propia.

La gravedad de la crisis de 2008 afectó a todos los ámbitos, y en la tabla anterior se observa que antes de comenzar a salir de la recesión (primer trimestre de 2014) más de 1,3 millones de ocupados estudiaban al mismo tiempo que trabajan. Pero conforme la situación económica iba mejorando, los 
trabajadores iban paulatinamente dejando de compatibilizar estudio y empleo, hasta que en septiembre de 2017 casi un 31\% menos de trabajadores cursaban estudios (reglados o no) en comparación a tres años y medio antes, bajando la cifra total del millón de personas.

El termómetro de la evolución de los puestos de trabajo tras la salida de la crisis es uno de los indicadores que mejor muestra la relación entre el esfuerzo por obtener educación superior y su vinculación con el empleo, tal y como se observa en la Tabla-19:

Tabla 19. Ocupados por nivel de formación, sexo y grupo de edad.

\begin{tabular}{|c|c|c|c|c|c|c|c|}
\hline \multirow{2}{*}{ (miles de personas) } & \multirow[b]{2}{*}{ 2017T3 } & \multirow[b]{2}{*}{$2016 \mathrm{~T} 1$} & & \multicolumn{3}{|c|}{2017 vs 2014} \\
\hline & & & $2015 T 1$ & 2014T1 & $\%$ & Diferencia & $\begin{array}{c}\text { Distribución } \\
\text { sobre total }\end{array}$ \\
\hline Total & $19.049,2$ & $18.029,6$ & $17.454,8$ & $16.950,6$ & $12,4 \%$ & $2.098,6$ & $12,4 \%$ \\
\hline Analfabetos & 37,1 & 50,0 & 39,8 & 41,5 & $-10,6 \%$ & $-4,4$ & $-0,2 \%$ \\
\hline Estudios primarios incompletos & 173,2 & 200,6 & 189,6 & 213,7 & $-19,0 \%$ & $-40,5$ & $-1,9 \%$ \\
\hline Educación primaria & 941,4 & 954,1 & 986,8 & $1.113,3$ & $-15,4 \%$ & $-171,9$ & $-8,2 \%$ \\
\hline $1^{\mathrm{a}}$ etapa ed.secundaria y similar & $5.223,7$ & $4.914,4$ & $4.744,2$ & $4.533,7$ & $15,2 \%$ & 690,0 & $32,9 \%$ \\
\hline $2^{\mathrm{a}}$ etapa ed.secundaria (orie.gral.) & $2.732,4$ & $2.534,0$ & $2.502,7$ & $2.362,3$ & $15,7 \%$ & 370,1 & $17,6 \%$ \\
\hline $2^{\mathrm{a}}$ etapa ed.secundaria (orie. prof.) & $1.843,0$ & $1.752,5$ & $1.637,3$ & $1.555,0$ & $18,5 \%$ & 288,0 & $13,7 \%$ \\
\hline Educación superior & $8.098,6$ & $7.624,0$ & $7.354,4$ & $7.131,1$ & $13,6 \%$ & 967,5 & $46,1 \%$ \\
\hline
\end{tabular}

Fuente: Instituto Nacional de Estadística - Elaboración propia.

De los 2,1 millones de nuevos empleos creados en España entre marzo de 2014 y septiembre de 2017 , casi la mitad han sido ocupados por titulados superiores (el $46,1 \%)^{3}$; y el número de ocupados con Educación Primaria o inferior descendió en 217.000 personas en el mismo periodo. Es decir, 1 de cada 2 nuevas oportunidades de empleo que el crecimiento económico experimentado por España ha puesto en la mesa del mercado de trabajo, han sido ocupados por titulados superiores (Gráfico-1).

Gráfico 1. Distribución de los 2,1 millones de nuevos empleos 2017 vs 2014 por nivel de formación (en miles de personas).

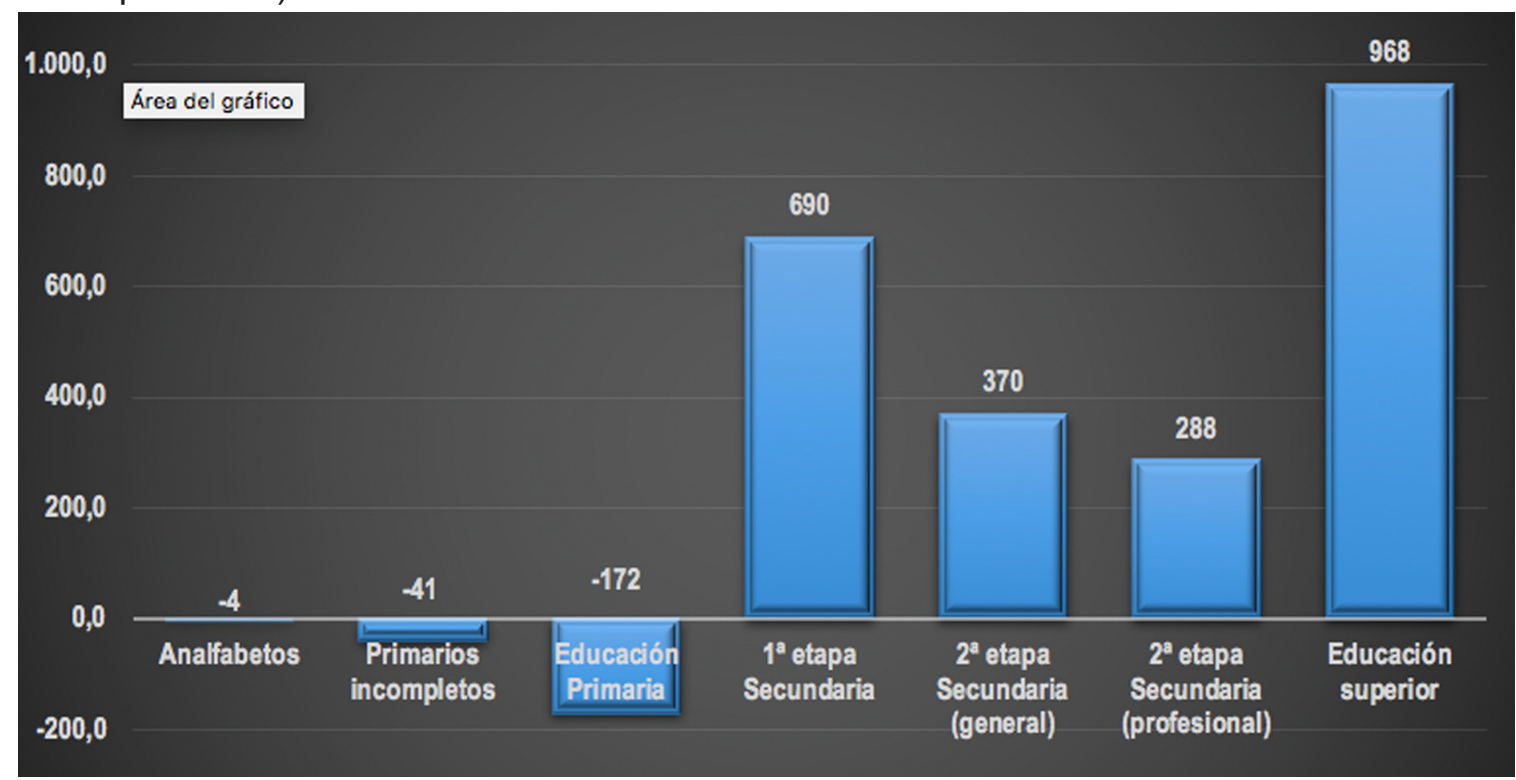

Fuente: Instituto Nacional de Estadística - Elaboración propia.

\footnotetext{
${ }^{3}$ Para una mayor calidad interpretativa de la Tabla-19 sería necesario descontar aquellos ocupados en el primer trimestre de 2014 que han seguido trabajando en el tercer trimestre de 2017 pero con un nivel formación superior, dato no disponible a la hora de realizar este trabajo pero que no desvirtúa la reflexión global realizada.
} 


\section{CONCLUSIONES}

El análisis realizado muestra que la universidad española es mayoritariamente pública, lo que no obsta para que el profesorado en centros privados sea considerablemente más joven y que los precios públicos para acceder a la educación superior se sitúen entre los más altos de Europa. Es probable que estos obstáculos sigan influyendo para que los principales trabajos de los españoles menores de 30 años sean en actividades de baja cualificación (camarero, dependiente en comercios y peón).

El gasto en educación en España ha descendido durante la crisis para el conjunto de las Administraciones, tanto la Administración General del Estado como las diecisiete comunidades autónomas. Pero este descenso ha sido inversamente proporcional al incremento del gasto realizado en educación por las familias, que no ha dejado de aumentar desde 2005.

Canarias ocupa las últimas posiciones tanto en inversión en I+D como en el porcentaje de su población que dispone de estudios superiores. Pero esta conclusión no sorprende al conocer que el gasto público del Archipiélago en enseñanza universitaria es considerablemente menor a lo que representa su población en el conjunto de España. No obstante, este marco global se matiza con el esfuerzo público realizado al becar a los alumnos, ya que en la Universidad de La Laguna hay un $53 \%$ más de alumnos becados que en la media de las universidades públicas españolas, y en la de Las Palmas de Gran Canaria un 35\% más.

Ambas universidades públicas canarias (especialmente la de La Laguna) están situadas entre las de mejor salud financiera del país, mostrando un menor endeudamiento y una mayor independencia financiera. Además, Canarias está situada en torno a la media nacional en cuanto a la tasa bruta de acceso a la universidad, y las plantillas de profesores de sus universidades públicas son más estables que la media del Estado; pero ello tampoco impide que tenga una baja tasa de escolarización universitaria, y que sólo Baleares ofrezca una peor tasa bruta de población que se gradúa.

Estas luces y sombras del panorama universitario canario, parecen indicar que aún no ha calado suficientemente entre la población las ventajas que ofrece realizar el esfuerzo necesario para obtener una educación superior, pues se mantienen porcentajes bajos de personas con formación universitaria pese a que estos ganan más dinero, colaboran más como voluntarios en proyectos sociales y tienen mayor participación en la política y en acciones de protesta y de movilización ciudadana.

Buena parte de los ocupados españoles mantuvieron una formación continua durante la crisis al mismo tiempo que trabajaban, probablemente porque temían perder su empleo o para mejorar su empleabilidad si eran despedidos; pero una vez que ha vuelto la bonanza económica, las personas que trabajan han comenzado a abandonar este hábito. Es decir, ha descendido el interés en la mejora de la formación de las personas con empleo durante el actual ciclo de crecimiento económico, cuando había aumentado considerablemente aquellos que continuaban formándose al mismo tiempo que trabajan durante la crisis iniciada en 2008.

El análisis realizado sobre el contexto de la enseñanza universitaria en España y Canarias en perspectiva comparada con el resto de los Estados miembros de la Unión Europea y de los países de la OCDE, ha permitido valorar los principales recursos existentes en el camino de la transición educativa hacia el empleo y concretar los resultados más importantes en ambos ámbitos territoriales. Y uno de los más destacados es la estrecha relación existente en España durante los últimos tres años entre el empleo y la cultura del esfuerzo para disponer de educación superior y titulación universitaria, ya que un millón de los 2'1 millones de empleos generados durante los tres últimos años, han sido ocupados por titulados universitarios. 


\section{REFERENCIAS BIBLIOGRÁFICAS}

Alegre, M., y Subirats, J. (2016). Sistemas y políticas educativas comparadas: transformaciones, convergencias y divergencias en los países europeos, en Del Pino, E., y Rubio M. J. (directoras), Los Estados de Bienestar en la encrucijada, 342-269, Madrid: Tecnos.

Comisión Europea (2017). Modernización de la Educación Superior en Europa: personal académico (Informe de Eurydice). Luxemburgo: Oficina de Publicaciones de la Unión Europea. Recuperado de: https://sede.educacion.gob.es/publiventa/descarga.action?f_codigo_agc=18753.

Conferencia de Rectores de las Universidades Españolas (2017). La Universidad española en cifras 2015/16. Recuperado de: http://www.crue.org/Documentos\%20compartidos/Publicaciones/Universidad\%20Espa\%C3\%B1ola\%20en\%20 cifras/UEC_15-16.PDF.

Instituto de la Juventud (INJUVE) del Ministerio de Sanidad, Política Social e Igualdad (2011). Desmontando a ni-ni: un estereotipo juvenil en tiempos de crisis. Recuperado de: http://www.injuve.es/sites/default/files/9206-01.pdf.

Instituto Nacional de Estadística (INE). Cifras de población. Recuperado de: http://www.ine.es/dyngs/INEbase/es/operacion. htm?c=Estadistica_C\&cid=1254736176951\&menu=resultados\&idp=1254735572981.

Instituto Nacional de Estadística (INE). Encuesta de población activa. Recuperado de: http://www.ine.es/dyngs/INEbase/es/ operacion.htm?c=Estadistica_C\&cid=1254736176918\&menu=resultados\&idp=1254735976595.

Ministerio de Educación, Cultura y Deporte (2016a). Datos y cifras del sistema universitario español. Recuperado de: https:// www.mecd.gob.es/dms/mecd/servicios-al-ciudadano-mecd/estadisticas/educacion/universitaria/datos-cifras/datosy-cifras-SUE-2015-16-web-.pdf.

Ministerio de Educación, Cultura y Deporte (2016b). Panorama de la educación. Indicadores de la OCDE 2016. Informe español. Recuperado de: http://www.mecd.gob.es/dctm/inee/eag/panorama2016okkk.pdf?documentld=0901e72b82236f2b.

OCDE. Education at a Glance 2016: OECD indicators. Recuperado de: https://www.keepeek.com//Digital-Asset-Management/oecd/education/education-at-a-glance-2016_eag-2016-en\#page1.

Requena, M., y Bernardi, F. (2008). El sistema educativo, en González, J.J., y Requena, M. (eds.), Tres décadas de cambio social en España, Madrid: Alianza Editorial.

Requena, M., Salazar, L., y Jonas, R. (2013). Estratificación social. Madrid: McGraw-Hill.

Servicio Público de Empleo Estatal (2016). Informe del Mercado de Trabajo de los Jóvenes. Recuperado de: http://www.sepe. es/contenidos/que_es_el_sepe/publicaciones/pdf/pdf_mercado_trabajo/imt2016_datos2015_estatal_jovenes.pdf.

Sodaro, M. J. (2010). Política y Ciencia Política: una introducción. Madrid: McGraw-Hil. 